\title{
REVIEW ARTICLE Pulmonary group 2 innate lymphoid cells: surprises and challenges
}

\author{
Malcolm R. Starkey ${ }^{1}$, Andrew NJ. McKenzie ${ }^{2}$, Gabrielle T. Belz ${ }^{3}$ and Philip M. Hansbro ${ }^{1,4}$
}

Group 2 innate lymphoid cells (ILC2s) are a recently described subset of innate lymphocytes with important immune and homeostatic functions at multiple tissue sites, especially the lung. These cells expand locally after birth and during postnatal lung maturation and are present in the lung and other peripheral organs. They are modified by a variety of processes and mediate inflammatory responses to respiratory pathogens, inhaled allergens and noxious particles. Here, we review the emerging roles of ILC2s in pulmonary homeostasis and discuss recent and surprising advances in our understanding of how hormones, age, neurotransmitters, environmental challenges, and infection influence ILC2s. We also review how these responses may underpin the development, progression and severity of pulmonary inflammation and chronic lung diseases and highlight some of the remaining challenges for ILC2 biology.

Mucosal Immunology (2019) 12:299-311; https://doi.org/10.1038/s41385-018-0130-4

\section{INTRODUCTION}

Innate lymphoid cells (ILCS) are a heterogeneous family of cells that include group 1 ILCs (ILC1s) characterized by their production of interferon- $\gamma($ IFN- $\gamma)$, ILC2s that predominantly express IL-5 and IL-13, and ILC3s that secrete IL-22 and/or IL$17 .^{1-3}$ ILC2s and ILC3s are important in maintaining tissue homeostasis by regulating lymphoid tissue development, tissue repair and fat metabolism. ${ }^{4-8}$ Collectively, ILCs protect the body against a multitude of organisms including intracellular pathogens, bacteria, parasitic worms and fungi. ${ }^{9-12}$ Nevertheless, when dysregulated they can promote chronic inflammation such as that which occurs in chronic obstructive pulmonary disease (COPD) driven by ILC1s and ILC3s or allergy and asthma that are promoted by ILC2s. Additionally, certain cancers are driven by ILC3s and some autoimmune diseases including inflammatory bowel disease and multiple sclerosis involve multiple ILC subsets. ${ }^{13-16}$ Here, we review recent advances in our understanding of the contribution of ILC2s in inflammation and immunity, with a particular focus on the lung, and the challenges in understanding their key roles in maintaining immune homeostasis and the implications for respiratory diseases and therapeutic intervention. We place this in the context of recent and surprising findings that have been enabled by the development of a wide range of molecular tools (Table 1) and ILC modulators (Table 2) and highlight some of the remaining challenges in ILC2 biology. ILC2s are typically investigated in relation to helminth infection and allergic responses. However, a recent explosion of data highlights multifaceted roles for these cells in immune responses and tissue homeostasis.
Transcriptional blueprint regulating ILC2 development

ILC2s are not a uniform population and there are inconsistencies in the markers they express. Nevertheless, ILC2s are delineated into at least two subsets: natural ILC2 (nILC2) that respond mainly to IL-33, and; inflammatory ILC2 (ilLC2) that are highly responsive to IL-25 $5^{17}$ and have the capacity to migrate between mucosal sites during inflammation in response to chemotactic signals. ${ }^{18}$ nILC2s are generally recognized by their expression of the IL-33 receptor (IL-33R, also known as ST2). ilLC2s express the activation marker KLRG1 and the IL-25R but strikingly, do not express ST2 which raises questions about the universal use of this receptor to mark ILC2s. ${ }^{19}$ It is proposed that ilLC2s are highly responsive precursors that are mobilized by inflammatory stimuli but ultimately adopt an nILC2-like, or ILC3-like phenotype. ${ }^{17}$

ILC2s are characterized by their expression of Gata binding protein-3 (Gata3) and their production of cytokines including IL-4, IL-5, IL-9 and IL-13. They arise from the common lymphoid progenitor (CLP) in the bone marrow which give rise to the more restricted ILC2 lineage-specific progenitor, ILC2p. The development of this progenitor relies on Gata3 ${ }^{20-23}$ and the transcription factor retinoic acid receptor-related orphan nuclear receptor-a (RORa encoded by Rora gene). ${ }^{24} \mathrm{~A}$ tight transcriptional network involving factors such as inhibitor of DNA binding-2 (Id2), Notch, ${ }^{25}$ nuclear factor interleukin-3 (Nfil3), ${ }^{26,27}$ promyelocytic leukemia zinc finger protein (PLZF, encoded by Btb16), T-cell factor-1 (TCF1), ${ }^{28,29}$ and zinc finger protein growth factor independent-1 $(\mathrm{G} f 1)^{30}$ are essential for the sequential specification and commitment of the ILC2 lineage. Indeed, TCF-1 acts through both Gata3dependent and -independent pathways to promote the

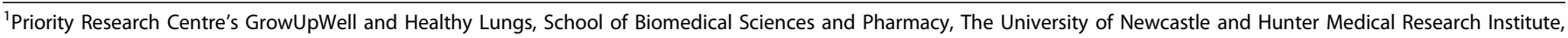
New Lambton Heights, NSW 2305, Australia; ${ }^{2}$ MRC Laboratory of Molecular Biology, Francis Crick Avenue, Cambridge Biomedical Campus, Cambridge CB2 0QH, United Kingdom;

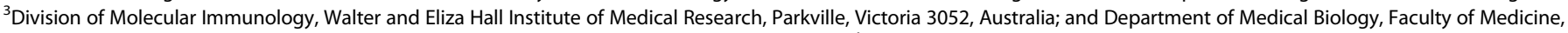

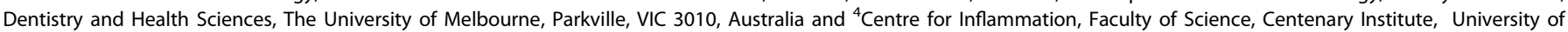
Technology Sydney, Ultimo, NSW 2007, Australia

Correspondence: Philip M. Hansbro (Philip.Hansbro@newcastle.edu.au)
}

Received: 30 October 2018 Revised: 18 December 2018 Accepted: 21 December 2018

Published online: 21 January 2019 
Table 1. Tools to explore ILC2 function

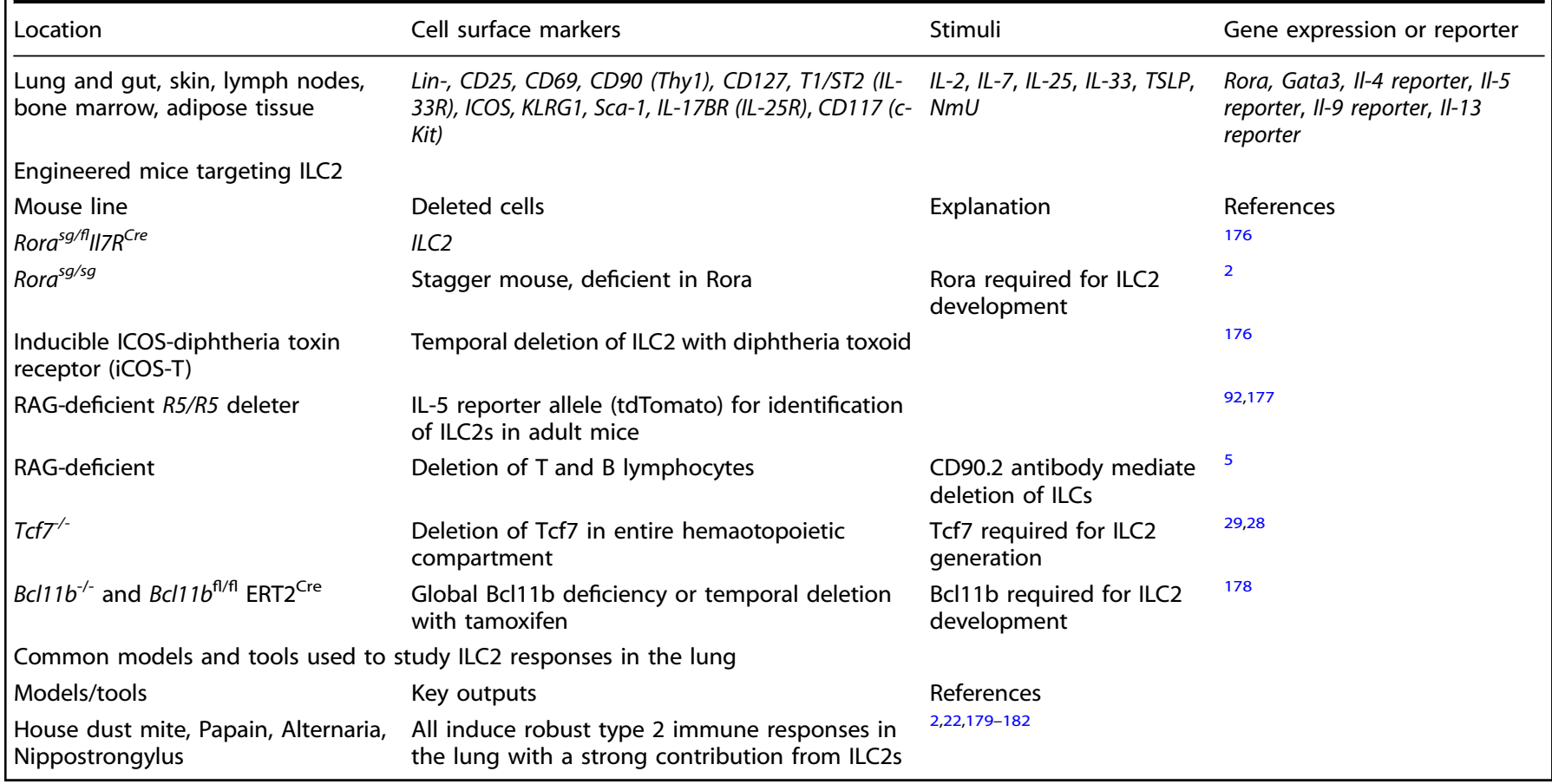

generation of $\mathrm{ILC} 2 \mathrm{~s},{ }^{29}$ but precisely how these two factors orchestrate the ILC2 programming is incompletely understood.

In adults, mature ILC2s are thought to originate from bone marrow progenitors and IL-33 promotes their egress. ${ }^{31-33}$ However, under certain circumstances, ILCs may also arise in the thymus where the levels of expression of transcription factors dictate the fate outcome of early T-cell progenitors to become either adaptive or innate immune cells. ${ }^{31,34}$ At least part of this program may be regulated by the transcriptional enhancer E-box proteins (E proteins) and Id proteins which modulate ILC2 levels. Indeed, overexpression of Id1 or the dual deletion of E2A and HEB results in hyper-inflammatory ILC2 responses following allergen challenge with papain and enhanced capacity to eliminate $N$. brasiliensis. ${ }^{31}$ Id 1 itself is not generally expressed by immune cells but overexpression of Id proteins, or removal of their $E$ protein binding partner, enhances Id activity and drives the development of cells that depend on it. ILC2 production can be generated in the thymus by culture with IL-7 and IL- $33^{24,25}$ suggesting that modulating the balance of transcription factors may determine an ILC fate outcome in vivo. ${ }^{31,34}$

Single cell ILC-omics uncovers the identity of the ILC2p ILCs exhibit considerable heterogeneity in terms of cell surface antigen and gene expression. This reflects differential responses that occur to continuous encounters with fluctuating stimuli at mucosal surfaces such as the lung and specializations in individual tissues. The capacity to trace ILC fate has been limited by the relative rarity of these cells, however, significant advances in genomics have enabled the detailed temporal mapping of the dynamic and quantitative contributions of transcriptional regulators in defining cellular trajectory.

Defining the earliest ILC progenitors has been elusive in both mouse models and humans. However, recent studies of small cell numbers and single cell analyses have uncovered new markers of early checkpoints in ILC development. Analysis of the common innate lymphoid progenitor (CILP) revealed that the dedicated ILC2 progenitor unexpectedly expressed the surface receptor programmed cell death protein 1 (PD-1), which in combination with IL-25R serves as a hallmark for this progenitor. ${ }^{35,36}$ Notably, the ablation of PD-1 in progenitors did not impact the development of ILC2p or ILC2s. ${ }^{35}$ PD-1 is a major target in immunotherapy and is an important negative regulator of effector gut KLRG1 ${ }^{+}$ILC2s (also known as ilLC2). ${ }^{17,37}$ The emergence of $\mathrm{KLRG}^{+}{ }^{+} \mathrm{ILC2} \mathrm{s}$ in the lung also appears to rely on PD-1. We speculate that differential regulation in distinct tissues could account for the higher abundance of $\mathrm{KLRG}^{+}{ }^{+} \mathrm{ILC} 2 \mathrm{~s}$ in the lung that are associated with inflammation and asthma. ${ }^{37}$ ILC2s also dynamically express the ligand PD-L1 during immune responses in the lung, and its ligation with PD- 1 on Th2 cells acts as innate regulatory checkpoint for the adaptive response leading to Gata3 and IL-13 upregulation by T-cells. ${ }^{38}$ Inhibiting PD-L1 or IL-13 during early-life bacterial infection of the respiratory system prevents more severe allergic airway inflammation in later-life. ${ }^{39,40}$ However, blockade of PD-L1 during the first two weeks postpartum in the absence of pathogenic infection maintained exaggerated responsiveness to HDM challenge of mice in early life. ${ }^{41}$ The expression of these two key checkpoint factors on ILC2s warrants a deeper dissection of the consequences of therapy as although this may augment anti-tumor immunity, autoimmune sequelae are potential unexpected outcomes of treatment.

Genome-wide probing of ILCs using RNA-seq, ChIP-seq, and ATAC-seq combined with mass cytometry enables multivariate mapping of transcriptional and epigenetic identity of thousands of gene profiles and regulatory elements in single cells with functional outputs such as cytokine expression and metabolic regulation. This has allowed the detailed exploration of regulatory circuits in mammalian cells. ${ }^{42-48}$ This type of detail challenged the simplified schema for ILCs previously proposed ${ }^{3}$ and suggests that there may be as many 15 different subcategories reflecting differences in tissue localization and the influence of various stimuli, including commensal bacteria, in shaping the epigenetic landscape of ILCs. Some subsets, however, exhibit characteristics typically associated with other or multiple subtypes. ILC2s are typified by their expression of Gata3, Hes1, Areg, II5 and II13 transcripts, a subclass of ILC2, ILC1/2, also express Gzma, Hopx and Epas1 normally associated with ILC1, while ILC2/3 produce $C x C 12$, 
Table 2. Potential ILC2 targets as novel therapies for chronic lung diseases

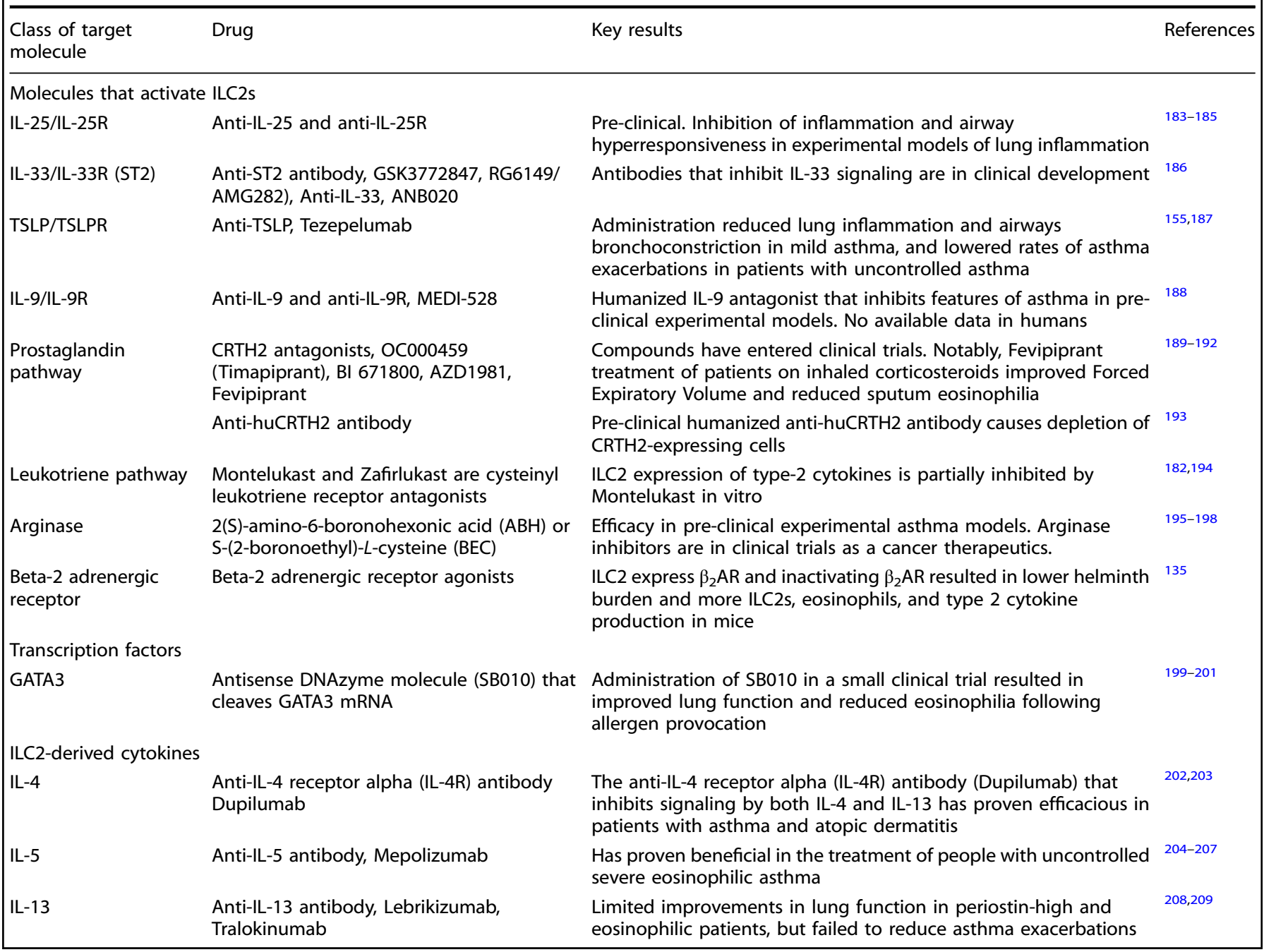

CxCl3 and Arg1 characteristic of ILC3. ${ }^{42}$ In all, four different subclasses of ILC2s were reportedly delineated. While these subsets all exhibited the signature gene Gata3, segregation into subsets was based on their graded expression of Gata3 and also KIf4, LIrg1, Ly6a and II2ra. The ILC2d subclass exhibited the highest levels of these genes, as well as $1 / 5$ and Csf2 and was distinguished from other subsets by their expression of Areg. These subclasses may result from heterogeneity introduced as a consequence of cues from their environment. This pattern was similar to that observed for T-bet suggesting that ILC subsets are not static, but their phenotypes reflect the dynamic equilibrium that occurs in implementing immune responses and maintaining homeostasis. The existence of these subclasses that do not neatly fit into the original proposed schema ${ }^{3}$ and imply that the plasticity reported by other groups in in vitro analyses are insights into such changes that can be induced in ILCs. Whilst this structure should be maintained it is a simplification of the numbers of subclasses that are encountered at different locations and during homeostasis or challenge. $^{49}$

The current classification of ILC subsets provides an important framework in which to view the development and functions of different ILCs. However, it is becoming clearer that the functional programs and capacity of ILC to respond to different settings is likely to be more complex. Functional plasticity, or the capacity to adopt phenotypes similar to other ILC subsets, provides the potential to fine-tune immune responses to different types of cytokines and enables the rapid adaption when confronted with pathogens (see also ref. ${ }^{50}$ ). Several studies have demonstrated this interconversion at least in vitro. Under the influence of IL-12 human Roryt ${ }^{+}$ILC3s convert into ILC1-like cells and are able to

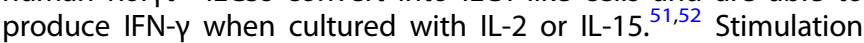
with IL-23 through the transcription factor STAT4 can also drive ILC3 conversion to ILC1s. ${ }^{53}$ In a separate report, IL-23 was shown to promote the reverse conversion of ILC1 into ILC3 although the molecular basis underpinning this pathway are not known. ${ }^{51}$ Similar pathways have also been shown in mice in which ILC3s can convert into IFN- $\gamma$-producing NK1.1 ${ }^{+}$ILC1 (also known as ex-ILC3s) and depends on the coordinate downregulation of Roryt. ${ }^{54}$ This pathway appears to be blocked in mice that lack T-bet ${ }^{55-57}$ or lose Notch2 or its downstream signaling molecule RBPj. ${ }^{45,58-60}$ ILC2s in both mice and humans can also adopt properties normally associated with ILC1s. ${ }^{13,14,61}$ Changes in ILC2s are largely driven by stimulation through the IL-1 $\beta$ receptor, and IL-1 $\beta$ is critical for inducing their proliferation and cytokine production. ${ }^{61}$ Human blood ILC2s have unstable surface phenotypes. ${ }^{62}$ Changes are potentiated by IL-13, IL-33 and IL-12 to generate IFN- $\gamma$-producing cells (also known as ex-ILC2s). This transition, like the ILC3 to ILC1 transition, also appears to depend on T-bet ${ }^{14,61,62}$ suggesting that the capacity to produce IFN- $\gamma$ depends on a common pathway in ILCs. Recently, transcriptional single cell profiling has revealed that there are also tissue-specific signals that imprint ILC2s and prime them for function. ${ }^{63}$ In this study tissue-specificity had a greater 
effect than even combined deficiency of IL-25, IL-33 and TSLP receptors. The tissue-specific transcriptome results in the expression of specific activation receptors, including in the skin. These events were not influenced by the microbiome shown using germ-free mice.

A comprehensive model describing how human ILCs develop, similar to that already undertaken in mice, has until recently been lacking, mainly as a result of technical limitations in probing rare immune populations. Recent advances in genomics and mass cytometry have, however, enabled the elucidation of these developmental steps in man. ${ }^{47,64,65}$ A striking initial finding identified that the transcriptional regulator RORyt (encoded by $R O R C 2$ in man) is not limited to ILC3s as found in mice but is broadly expressed in human ILC progenitors including those of ILC2. ${ }^{66}$ Lin $^{-} \mathrm{CD} 34^{+} \mathrm{CD} 45 \mathrm{RA}{ }^{+} \mathrm{CD} 117^{+} \mathrm{IL}-1 \mathrm{R} 1^{+} \mathrm{ID} 2^{+} \mathrm{RORyt}^{+}$progenitors were found in secondary lymphoid tissues and gave rise to all ILC subsets, but not other cell lineages. ${ }^{66}$ This raised the concept that differentiation could differ between the two species and that RORyt may play a potential role in humans distinct from that in mice. Aligned with this finding, although signature factors such as GATA3 were strongly associated with and most highly expressed in human ILC2s, this transcription factor was also expressed by other subsets such as ILC1s and ILC3s. ${ }^{65}$ Consequently, it appears that the $\mathrm{G}$ protein-coupled receptor CRTH2 (also known as $\mathrm{DP}_{2}$ ) is the most reliable marker for separating human ILC2s from ILC3s. This is also the case in the mouse where ILC1s and 3s are intermediate for Gata3 and ILC2s have high expression. $^{67,68}$

\section{Emerging anti-inflammatory role for ILC2s}

ILC2s are well known inducers of type-2 inflammatory responses, but recent evidence indicates that they are also involved in the resolution of inflammation and prevention of cell death through IL-9. ${ }^{69,70}$ ILC2s are the dominant source of IL-9 during the resolution of arthritis and IL-9 $9^{+}$ILC2s were located in close proximity to regulatory $\mathrm{T}$ (Treg) cells in inflamed synovium. ${ }^{69}$ In the absence of IL-9, ILC2-induced proliferation and activation of Tregs was impaired leading to chronic arthritis. In contrast, treatment with rIL-9 promoted ILC2-dependent Treg activation and effectively resolved inflammation. ${ }^{69} \mathrm{~A}$ more recent study demonstrated that artificially increasing ILC2s significantly attenuated experimental arthritis in an IL-4/13-dependent manner. ${ }^{71}$ Clinical relevance was demonstrated in remitting rheumatoid arthritis patients who had high numbers of ILC2s and IL-9 $9^{+}$ILC2s in their joints and circulation that inversely correlated with disease severity ${ }^{69,71}$ In a mouse cecal and ligation puncture model, sepsis induced IL-33 release and subsequent expansion of IL-9-secreting ILC2 in the lung, which prevented lung endothelial cell pyroptosis by attenuating caspase- 1 activation. ${ }^{70}$ Autocrine production of IL-9 supports ILC2 responses that co-ordinate epithelial cell maintenance and lung homeostasis. ${ }^{72}$ Collectively, these data indicate a pivotal role for ILC2s in resolving chronic inflammation, and the potential for their therapeutic manipulation. ${ }^{69}$ Further exploration of their anti-inflammatory capacity in other tissues and diseases is required.

\section{Bidirectional relationships between ILC2s and Tregs}

A complex bidirectional relationship exists between pulmonary ILC2s and Tregs. Transient depletion of Tregs induced IL-2- and CD25-dependent proliferation of ILC2s, suggesting that ILC2s can directly access IL-2 in the lung and that Tregs restrain the IL2-dependent expansion of these tissue-resident ILCs. ${ }^{73}$ Human and murine ILC2s express both ICOS and ICOS-ligand (ICOS-L) and ICOS:ICOS-L interactions on these cells promotes type-2 cytokine production and ILC2 survival through STAT5 signaling. ${ }^{7,75}$ A lack of ICOS on murine ILC2s reduced airway hyperresponsiveness (AHR) and lung inflammation, and blocking ICOS:ICOS-L interactions in human ILC2s suppressed their pro-inflammatory effects. ${ }^{74}$
Induced Tregs (iTreg) but not natural Tregs, inhibited the production of ILC2-drived IL-5 and IL-13 in vitro and in vivo. ${ }^{76}$ iTreg mediated suppression of ILC2s required ICOS-ICOS-Ldependent cell-to-cell contact in addition to the suppressive cytokines TGF- $\beta$ and IL-10. ${ }^{76}$ Human iTreg can suppress human ILC2s through ICOSL to control airway inflammation in a humanized ILC2 mouse model. ${ }^{76}$ Furthermore, human ILC2s express IL-10RA and TGFBR2 receptors and the addition of rIL-10 or TGF- $\beta$ to ex vivo ILC2 cultures suppressed type- 2 cytokine production. ${ }^{77}$ In contrast, TGF- $\beta$ is also a chemoattractant for ILC2s that express TGFBR2 that contributes to pulmonary responses to allergen. $^{78}$

Other cytokines such as IFN- $\gamma$ can suppress IL-33-induced ILC2 activation and control Treg numbers and type-2 responses. ${ }^{75}$ ILC2s express the IFN- $\gamma$ receptor ${ }^{44}$ and IFN- $\gamma$ directly represses ILC2 activation, cytokine production and proliferation in vitro. ${ }^{75}$ In vivo IL-33-induced lung ILC2 proliferation and accumulation was blocked by co-administration of IFN- $\gamma$ and mice that overexpress IFN- $\gamma$ had fewer ILC2s. Listeria monocytogenes infection, which elicits potent IFN- $\gamma$ responses, also suppressed ILC2 function in an IFN- $\gamma$-dependent fashion. ${ }^{75}$ OX40L expression by ILC2s is also required for IL-33-driven Treg and Th2 cell expansion. ${ }^{79}$ Collectively, these studies highlight a multifaceted interplay between ILC2s and Tregs and the factors that regulate their function.

\section{New influencers of ILC2 function}

The immune system is subject to both environmental and intrinsic influences. Recent advances highlight the importance of circadian rhythm, sex hormones, age and neurotransmitters in regulating ILC2 function, especially in the lung.

\section{Circadian rhythm}

The circadian clock has emerged as an important factor influencing the efficiency of immune response generation. ${ }^{80}$ Its daily oscillations are mediated principally via the suprachiasmatic nucleus which entrains peripheral body clocks. ${ }^{81,82}$ In mammals, this clock depends on highly conserved transcriptional regulators such as CLOCK, BMAL1 and REV-ERBa nuclear proteins that bind to E-box sequences. The circadian clock involves many other genes such as Id2 and the repressor nuclear factor interleukin-3 (NFIL3, also known as E4BP4), which are also associated with the immune system and influence the core and secondary feedback loops involving Rev-ERBa. Rora, a critical transcription factor for ILC2 development, ${ }^{24}$ is an activator of Bmal1 transcription within the suprachiasmatic nucleus. ${ }^{83}$ It is required for normal BMAL1 expression and consolidation of daily locomotor activity and is regulated by the core clock in the suprachiasmatic nucleus. This suggests that opposing activities of Rora and REV-ERBa, are important in the maintenance of circadian clock function. ${ }^{83}$ NFIL3 is required for the development of the ILC progenitor, ${ }^{26,27}$ which may be independent of the cellular clock, however, it also regulates Th17 cells by linking their development to the circadian network though REV-ERBa. ${ }^{84}$ REV-ERBa represses proinflammatory cytokine production and controls the amplitude of pulmonary inflammation to inhaled endotoxin. ${ }^{85,86}$ Similarly, BMAL1 regulates the diurnal rhythms of inflammatory monocytes facilitating their mobilization in immune defense. ${ }^{87}$ This effect is at least partially mediated through inhibiting NF-KB activation and the induction of miR-155. ${ }^{88}$ miR-155 deletion ablates the circadian rhythmicity of cytokine production and leads to increased susceptibility to lipopolysaccharide-induced sepsis. miRNAs are key regulators of ILC2-induced allergic inflammation and miR-155 is increased in ILC2s in response to stimulation with IL-33. Moreover miR-155 $5^{-/-}$ mice have reduced IL-33-induced ILC2 proliferation and cytokine production..$^{89,90}$ Tissue ILC2s regulate eosinophilopoiesis and accumulation in tissues through constitutive cytokine production, particularly IL-5. They also express the vasoactive intestinal peptide receptor type-2 (VPAC2) which responds cyclically and 
potentially provides a mechanism by which ILC2 are directly linked to circadian and metabolic rhythms and maintain eosinophil homeostasis. ${ }^{91,92}$ Collectively, multiple facets of the immune system are influenced by the circadian clock and factors critical for the development and maintenance of ILCs are implicated in secondary regulation of circadian oscillations. Further experimental confirmation of these observations is warranted.

\section{Sex hormones}

The prevalence of certain diseases may differ in incidence and severity between males and females. Men are more susceptible than women to developing severe asthma in childhood, however, following puberty, this trend reverses with females having the highest incidence of allergies in adulthood, ${ }^{93,94}$ which correlate with higher circulating ILC2 numbers. ${ }^{95}$ The mechanisms underlying these differing susceptibilities has been elusive but the patterns of pathogenesis implicate sex hormones. Several immune cell lineages, including myeloid cells and lymphocytes express receptors for estrogen, progesterone and androgens (testosterone, dihydrotestosterone and androstenedione) and are hormone regulated and influence both innate and adaptive immune responses. ${ }^{96}$ In macrophages, estrogen receptor signaling inhibits the production of NF-KB-regulated proinflammatory genes such as IL- 6 while the activation of ERa46 impairs leukocyte migration by inhibiting CCL2 expression. ${ }^{97,98}$ Similarly, in dendritic cells (DCs), estrogen-dependent activation of ERa regulates the development and/or functional responses of particular subsets. ${ }^{99,100}$ Progesterone also has both stimulatory and suppressive roles in immunity. Progesterone receptors are expressed principally by T-cells, including Tregs and NK cells, in addition to DCs and mesenchymal stem cells. ${ }^{101-103}$ Early studies demonstrated the impact of hormones on Th2 immunity. Hepworth et al., showed the effects of sex hormones on Th2 populations, analogous to ILC2s, during helminth infection. ${ }^{104}$ Female estradiol increased the induction of Th2 responses in vitro but was not critical for these responses in mice. Male testosterone suppressed DC-induced T-cell stimulation, immune responses and Trichuris muris clearance that was restored by castration. The effects may be though IL-18 induced suppression of Th2 responses. In NK cells, progesterone downregulates the secretion of IFN- $\gamma$, dampening down uterine NK cell function. ${ }^{105}$ The active metabolite of testosterone is dihydrotestosterone which irreversibly binds to the androgen receptor (AR). ${ }^{106}$ The AR is expressed at various levels by a variety of leukocytes such as neutrophils and macrophages. ${ }^{107}$ Most recently, the AR was identified on ILC2s and signaling through this receptor reduced the susceptibility to IL-33-driven and alternaria extract-driven lung inflammation in part by reducing the expansion and reactivity of ILC2s. ${ }^{95,108}$ Signaling through the AR pathway provides support for the protective role of androgens in allergic asthma and the dimorphic switch that occurs after puberty. The lungs of female mice harbor significantly greater numbers of ILC2s during homeostasis, mostly due to the presence of KLRG $1^{-}$ILC2s that are largely absent in male lungs. ${ }^{109}$ These KLRG $1^{-}$ILC2s are capable of producing type-2 cytokines and increased with age and sexual maturity, suggesting the existence of a unique functional ILC2 subset in females. The frequency of $\mathrm{PLZF}^{+}$ILC precursors were higher in males and further increased by androgens, suggesting that male sex hormones inhibit the conversion of ILC precursors to ILC2s. However, these sex dependent effects appear to be specific for tissue location and disease context. In contrast to the lung, adult male mice have increased ILC2 numbers driven by mast cell-derived IL-33 in the central nervous system (CNS), which is an immune-protective mechanism and may contribute to the sexual dimorphism observed in the demyelinating disease multiple sclerosis to which females are more susceptible. ${ }^{10,111} \mathrm{IL}-33$ responsive ILC2s are also present in the uterus and regulated by estrogen, however, lung
ILC2s were not altered by estrogen administration or in ovariectomized mice. ${ }^{112}$

Age

The first breath and resulting inflation of the lung triggers IL-33 production by type II alveolar epithelial cells and induces the expansion of ST2 ${ }^{+}$ILC2s in newborn mice. ${ }^{13,114}$ Consequently, few $\mathrm{ST}_{2}{ }^{-}$ILC2s are present in the lungs embryonically and in newborn mice, but numbers are markedly increased by postnatal week 1 , peak during postnatal week 2 to levels three times that of adult mice and then decrease and stabilize by week $6 .^{92,114-116}$ However, the mechanisms responsible for ILC2 contraction are incompletely understood. ${ }^{115}$ Interestingly, neonatal mice had fewer ILC2s in their liver and small intestine compared to adults, suggesting that the functional relevance of increased ILC2s in early-life is likely limited to the lung. ${ }^{114}$ Importantly, a significant proportion of neonatal lung and draining mediastinal lymph node ILC2s co-expressed intracellular IL-5 and IL-13 and had increased proliferative capacity compared to adult ILC2s in vitro. ${ }^{114}$ This is in contrast to adult lung ILC2s that appear to be only constitutive IL5 producers, with these IL- $5^{+}$ILC2s found embedded in collagenrich regions near the confluence of medium-sized blood vessels and airways but absent from the alveolar structures. ${ }^{2,92}$ However, the precise location of ILC2s in the developing neonatal lung is unknown, and they may reside in the alveolar compartment. Furthermore, neonatal lung ILC2s have different cell surface antigen expression with less CD90.2 and CD25 than adult lung ILC2s, but more ST2 and comparable intracellular GATA3 levels. ${ }^{116}$ Collectively, these data indicate phenotypic and functional differences between neonatal and adult ILC2s in the lung, suggesting that their impact in lung development is not just related to ILC2s numbers.

Early-life Th2-associated immune skewing and susceptibility to allergy are often considered remnants of feto-maternal symbiosis. ${ }^{115}$ Indeed, neonates are prone to sensitization by allergens and Th2 cell-driven allergic disease as their immune system is Th2biased. ${ }^{117,118}$ The IL-33-dependent expansion of ILC2s during the neonatal period may be important in the reportedly exaggerated HDM-induced AHR in newborns. ${ }^{41}$ During the alveolarization phase of postnatal lung development, HDM exposure further increased IL-33, which increased cytokine production by ILC2s and activation of CD11 b ${ }^{+}$DCs driving greater Th2 skewing. ${ }^{115}$ These data indicate that the alveolarization period has type- 2 dominant immunity with exaggerated innate immune responses to allergens. This may promote Th2-skewed immune responses that may explain the increased asthma prevalence in childhood.

The structural features of postnatal lung development which consists mainly of the formation and remodeling of alveoli are similar between humans and mice, despite obvious timeframe differences. The most active phase of alveolar development occurs during the second postnatal week in mice and between years 2-3 in humans. The discovery of substantially increased IL-33 production and accumulation of ILC2s during the alveolarization phase of lung development raises the question of why a spontaneous IL-33-dependent type- 2 immune cell microenvironment evolved in mammalian lungs. It is tempting to speculate that type-2 immunity controls lung development or remodeling of the lung postnatally. Indeed, IL-33 is known to activate alternatively activated M2 macrophages that control tissue remodeling and postnatal branching morphogenesis of the lung. ${ }^{13,119,120}$ However, despite this no gross abnormalities in lung alveolarization were observed in IL-33-deficient mice. ${ }^{113,115}$ It has been suggested that ILC2s may also be involved in lung regeneration and may be a potential immunomodulatory target to stimulate alveologenesis in adult mice. ${ }^{121}$ ILC2s and lung macrophages modulate the regenerative microenvironment to support alveolar epithelial stem cell proliferation and differentiation. ${ }^{121}$ IL-5 $5^{+}$ILC2s were found near small airways and in alveolar spaces post 
pneumonectomy, however, no direct studies were performed indicating the requirement for ILC2s in lung regeneration or alveolarization. ${ }^{121}$ T-cell and B-cell deficient mice had normal lung mass indicating that adaptive immunity is unlikely to play a role in lung regeneration, highlighting the importance of innate immunity and possibly ILC2s. ${ }^{121}$ Indeed, ILC2s have also been shown to promote lung tissue homeostasis after infection, as well as disrupting bronchial epithelial barrier integrity in asthmatic patients. $5,72,122$ Thus, the role of ILC2s in lung development, repair and homeostasis is complex and remains to be fully elucidated.

\section{Neurotransmitters}

Initial evidence that neurotransmitters may modulate immune responses was that their release from nervous tissue could lead to signaling through lymphocyte cell surface receptors. ${ }^{123}$ Leukocytes express receptors for the main brain neurotransmitters such as glutamate, dopamine and serotonin, ${ }^{123}$ and release neurotransmitters that act as autocrine or paracrine modulators. ${ }^{124}$ Neuromedin $U$ (NMU) is a neuropeptide expressed by the CNS, but also various peripheral organs including the lung and gastrointestinal tract, where ILC2s are abundant. Very recently, ILC2s have been identified in the mouse CNS. ${ }^{110,125} \mathrm{NMU}$ interacts with two G protein-coupled receptors, NMU-R1 and NMU-R2. NMU-R2 is expressed in a specific region of the brain and NMU-R1 is expressed in various peripheral tissues, including immune and hematopoietic cells. ${ }^{126}$ Early work demonstrated that NMU is involved in type-2 immune responses including mast cellmediated inflammation, activation of murine and human eosinophils and allergen-induced lung eosinophilia. ${ }^{127,128}$ However, the underlying mechanisms remain elusive. A recent triad of work demonstrate a clear role for NMU in activating mucosal ILC2s and type-2 inflammation. ${ }^{129-131}$ Profiling of lung resident ILCs from mice at baseline and after stimulation with IL-25 or IL-33 using parallel droplet-based SCRNA-seq, identified the neuropeptide receptor Nmur1 as a novel ILC2-specfic gene that was selectively expressed by mature ILC2 and ILC2p, but not other hematopoietic cells at baseline or after IL-25 stimulation. ${ }^{129-131}$ ILC2s also colocalized with cholinergic neurons that express NMU. ${ }^{129}$ NMU activated ILC2s in vitro, and administration of NMU alone induced lung inflammation ${ }^{129}$ and when co-administered with IL-25, substantially increased allergic airway inflammation by expanding ilLC2s. ${ }^{129,130}$ Furthermore, administration of NMU in vivo induced potent type- 2 cytokine responses that resulted in accelerated expulsion of $N$. brasiliensis. ${ }^{29}$ This was supported by autonomous ablation of Nmur1 in ILC2 that resulted in poor worm control. ${ }^{131}$ Loss of NMU-NMUR1 signaling reduced ILC2 frequency and effector function and altered transcriptional programs after HDM challenge in vivo. ${ }^{130}$ Interestingly, NMU expression in asthmatic bronchial brushings correlates with disease severity, ${ }^{132}$ which could be related to NMU-mediated ILC2 activation. These studies raise the question of whether NMU potentiates airway inflammation when high levels of innate type- 2 cytokines like IL-25 are present after virus-induced asthma exacerbations. ${ }^{133}$ These data indicate that NMU-NMRU1 signaling provides a selective mechanism that integrates the enteric nervous system and innate immune system to induce rapid type- 2 immune responses at mucosal sites. $^{129}$

Another mechanism of neuronal-associated ILC2 activation involving a rare airway epithelial cell population known as pulmonary neuroendocrine cells (PNECs) has been recently shown to act through calcitonin gene-related peptide to stimulate ILC2s. ${ }^{134}$ PNECs also act through the neurotransmitter gammaaminobutyric acid (GABA) to induce mucus secreting-cell hyperplasia in the airways, and the lungs of human asthmatics had increased PNECs. PNECs reside in close proximity to ILC2s and can stimulate ILC2 cytokine production and likely form neuro-immune modules at airway branches to amplify allergic asthma. ${ }^{134}$ Moreover, certain neuronal cues suppress ILC2 function. Murine
ILC2s express the $\beta_{2}$ adrenergic receptor $\left(\beta_{2} A R\right)$ and colocalize with adrenergic neurons in the intestine. ${ }^{135} \beta_{2} A R$ deficiency resulted in exaggerated ILC2 responses and type- 2 inflammation in the intestine and lung. Conversely, $\beta_{2} A R$ agonist treatment impaired ILC2 responses and reduced inflammation in vivo. Mechanistically, the $\beta_{2} A R$ pathway is a cell-intrinsic negative regulator of ILC2 responses through inhibition of cell proliferation and effector function. This study provides the first evidence of a neuronal derived regulatory circuit that limits ILC2-dependent type-2 inflammation. ${ }^{135}$ Given the importance of ILC2s in driving type- 2 immune responses it appears that $\beta_{2} A R$ may function as a molecular rheostat to fine-tune ILC2 responses and prevent pathologic type-2 inflammatory responses. These studies also highlight the contrasting functions of adrenergic and cholinergic neurons in regulating ILC2 function. These opposing functions appear to have evolved in the mammalian nervous system as a dual mechanism to rapidly repress or activate ILC2s to protect the host against diverse inflammatory stimuli. ${ }^{135}$

ILC2s are innate gatekeepers of respiratory immunity and homeostasis in chronic lung diseases and infection

Emerging evidence strongly implicate ILCs in the pathogenesis of chronic respiratory inflammation and diseases including asthma, COPD, pulmonary fibrosis and cystic fibrosis. ILCs also regulate immune responses and restoration of lung homeostasis following respiratory infections, and are involved in the infectious induction, exacerbation and onset of severe phenotypes of chronic respiratory diseases. Defining the roles of ILC2s in respiratory disease will clarify their use as diagnostic markers and their manipulation will highlight their potential for therapeutic targeting in disease prevention (Table 2). There have been few studies of human ILCs and additional studies are clearly warranted.

\section{ILC2s are critical mediators of allergic airway inflammation and asthma}

The airway epithelium is a crucial barrier enabling transport of gases and molecules whilst protecting from environmental challenges, inhaled particles and pathogens. In the healthy state ILCs are present in low numbers in the submucosa and maintain tissue homeostasis, but they are early immune responders and mediate tissue repair in response to challenge, and can also drive airway inflammation and chronic respiratory diseases. ${ }^{136}$ ILC stimulation depends on their microenvironment and activation by cytokines, lipids, microbes and their metabolites and contact with other cells in the respiratory tract. ${ }^{137}$

Allergic asthma is an archetypal type-2 immune-mediated airway disease that typically develops in childhood, often consequent to bronchiolitis or wheezing induced by respiratory viral (respiratory syncytial virus [RSV], rhinovirus [RV]) or bacterial (Chlamydia, Mycoplasma) infections. ${ }^{138-140}$ It is also exacerbated by these and influenza $A$ virus (IAV) infections, which may drive more severe type-1/-17 associated disease. ${ }^{141-143}$ Polymorphisms in IL-33, ST2, RORa and IL-13 are associated with type-2 high asthma and are critical for ILC2 development and activation. ${ }^{144}$ Recent epigenome analysis links gene regulatory elements around these loci in ILC2s to asthma susceptibility, ${ }^{145}$ which further supports the role of these genes in asthma. IL-33 and ILC2s are elevated in the airways and blood of asthma patients, ${ }^{146,147}$ and along with the levels of IL-5 and IL-13, increase with asthma severity. ${ }^{147-149}$

The mechanisms of induction of ILC2 responses that drive asthma are beginning to be unraveled and involve innate type- 2 cytokines and metabolic changes (Fig. 1a). Heterogeneity occurs in response to different stimuli with IL-33 inducing a uniform ILC2 phenotype, whereas HDM-induced ILC2s were heterogeneous but could still induce high levels of Th2 cytokines. ${ }^{22}$ Transcriptional profiling showed 1,600 differentially expressed genes in BAL ILC2s involved in adaptive $B$ and $T$ cell responses, proliferation and 

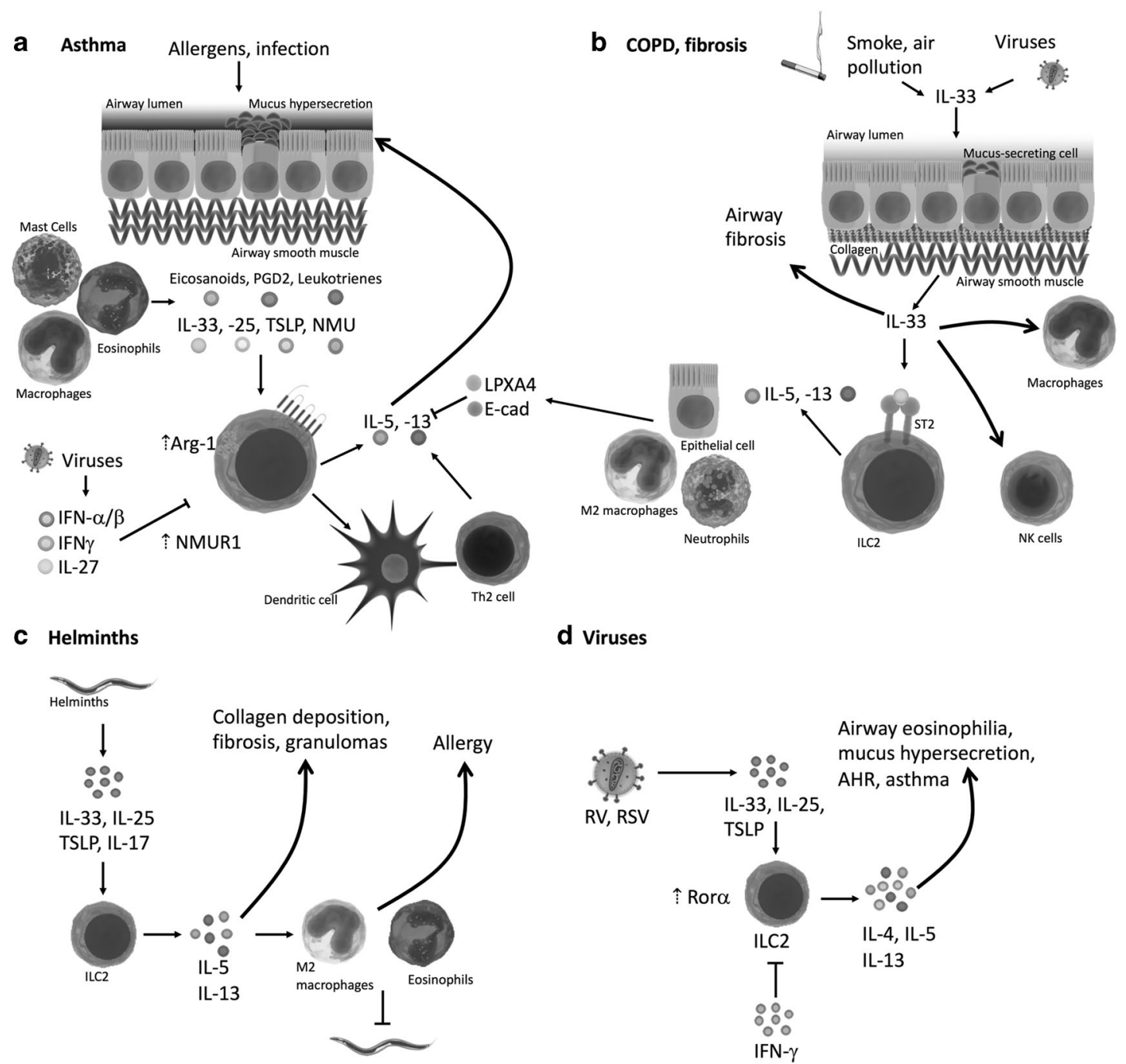

\section{d Viruses}

e Virus-induced exacerbations of asthma \& COPD

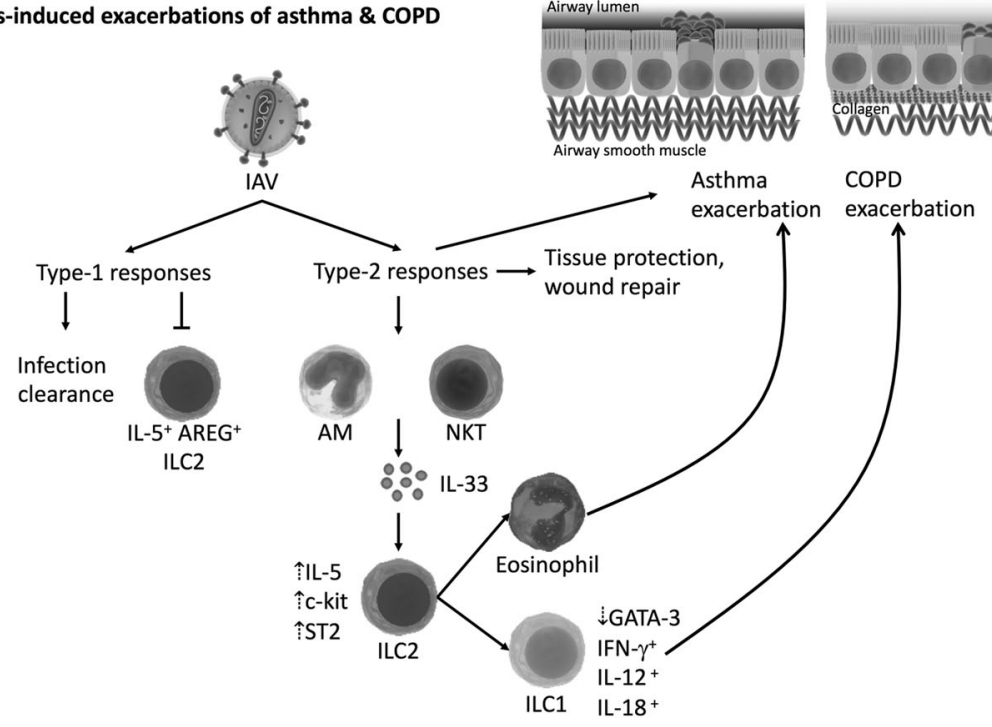

cytokine production. ILC2s can also acquire a memory phenotype during allergic airway inflammation. ILC2s persist after allergic inflammation has resolved and potently respond to unrelated allergens promoting exaggerated disease. ${ }^{150}$ These "memory"
ILC2s have a similar transcriptional profile to memory T cells. ILC2s respond to eicosanoids, prostaglandin D2 and leukotrienes produced by mast cells, macrophages and eosinophils. Important to asthma pathogenesis, with or without co-stimulation with IL-25 
Fig. 1 ILCs in respiratory diseases. a In asthma ILC2 development and activity is driven by eicosanoids, prostaglandin D2 (PGD2) and leukotrienes from mast cells, macrophages and eosinophils, as well as innate type-2 cytokines IL-33, IL-25, TSLP and neuromedin U (NMU). This is accompanied by increases in ILC2 markers such as Arginagse- 1 and the neuropeptide receptor Nmur1. Activated ILC2s release IL-5 and -13 that activate DCs and Th2 cells to reinforce type-2 immunity and asthma pathogenesis by inducing eosinophilic airway inflammation, mucus hypersecretion, airway remodeling and airway hyperresponsiveness (AHR, wheezing). These responses are opposed by lipoxin A4 and Ecadherin actions released from neutrophils, epithelial cells and macrophages, and by increases in type I IFNs, IFN- $\gamma$ and IL-27 that are induced by typical viral infections. b In COPD cigarette smoke and likely air pollution induce the increased expression of IL-33 that is released upon viral infection and exacerbates the underlying disease. However, acute smoke exposure reduces IL-5 and IL-13 responses and ST2 expression on ILC2s. ST2 inhibition suppresses virus-induced exacerbation of acute cigarette smoke-driven inflammation, IL-33 enhances macrophage and NK cell killing of virus, and ILC2s and IL-33 promote airway fibrosis. c. Helminth infections induce granulomas and fibrosis that are dependent on IL-33, IL-25, TSLP, IL-17 and IL-13 ${ }^{+}$ILC2s. Combined targeting of IL-33, IL-25 and TSLP suppressed type-2 driven inflammation, $\mathrm{IL}-13^{+} \mathrm{ILC} 2 \mathrm{~s}$, airway remodeling and fibrosis. IL-5 $5^{+}$and IL-13+ $\mathrm{ILC} 2 \mathrm{~s}$ induce macrophage, eosinophil and mucus activity that destroy and clear helmniths but eosinophils promote fibrosis and allergy. $\mathbf{d}$ In early life, rhinovirus (RV) and respiratory syncytial virus (RSV) induce IL-33, IL-25, TSLP and IL-13+ ILC2s, airway eosinophilia, mucus hypersecretion and AHR and predispose to the development of asthma. IFN- $\gamma$ suppresses ILC2s, but infection reduces IFN- $\gamma$, IL-12 and TNF expression. e Asthma and COPD patients are more susceptible to bacterial and viral infections such as with influenza A virus (IAV), which induce the accumulation of ILC2s in the lung that are tissue protective. They also typically induce type-1 responses that restrict ILC2s but also type- 2 responses that are responsible for tissue protection but IL-13 and -33 contribute to asthma exacerbations. IAV infection induces AHR independently of Th2 cells in an IL-13/-33/ST2/ILC2-mediated axis. IAV infection induces IL-33 production from alveolar macrophages and NKT cells that increase ILC2s, and causes transient IL-5 production, and the influx of c-kit ${ }^{+}$ST2 $^{+}$ ILC2 $s$ and associated eosinophils that exacerbate asthma. In COPD ILC2s are plastic and upon IAV infection acquire an ILC1 phenotype, with reduced GATA-3 expression and produce IFN- $\gamma$, IL-12 and IL-18. These cells reinforce virus-induced inflammation and exacerbations

or IL-33, ILC2s produce IL-4 and IL-13, activate and mobilize DCs and their release of Th2-cell recruiting CCL17, present antigen through MHC-II, and drive Th2 responses including IL-4, IL-5 and IL-13 production that is independent of antigen. ${ }^{151,152}$ Conversely the pro-resolving mediator lipoxin A4 and E-cadherin (ligand for KLRG1) from neutrophils, epithelial cells or $M 2$ macrophages reduce cytokine production by ILC2s. ${ }^{151,152}$ Recent studies showed that the expression of the intracellular metabolic factor arginase-1 (Arg-1) increased in mouse and human ILC2s during acute and chronic lung inflammation. ${ }^{153}$ This occurred through the inhibition of Arg-1 enzyme activity that disrupted numerous ILC2 metabolic processes including altered arginine catabolism and reduced polyamine biosynthesis and aerobic glycolysis. ${ }^{153}$ Collectively these data show that type-2 immune factors drive ILC2 development and metabolic factors regulate ILC2 activity that may promote asthma development. Furthermore, the roles in other phenotypes of asthma, including severe steroid resistant forms, which are often associated with respiratory infections, remain to be resolved and could be elucidated using mouse models that recapitulate the hallmark features of the human disease, as well as human tissues and cells. ${ }^{142,143,154}$ These murine studies are being translated into human therapies. To date, treatment with antibodies against IL-33 and TSLP suppressed airway inflammation and AHR after allergen challenge, and anti-prostaglandin D2 (PGD2) receptor (anti-CRTH2 antagonist) improved asthma control and lung function ${ }^{155,156}$ (Table 2).

\section{ILC2s in other chronic respiratory diseases}

There is a paucity of information on the roles of ILCs in the pathogenesis of other chronic respiratory diseases. ILC2 numbers are lower in the lung tissue of severe COPD patients compared to mild COPD or healthy controls but the numbers of total $\mathrm{CD} 45^{+}$ lymphocytes were not altered. ${ }^{13}$ An important study by Kearley et al., ${ }^{157}$ in COPD patients indicated that increased IL-33 levels occur that correlated with reduced lung function (FEV1), and its production was induced by viral but not Alternaria infection of human bronchoepthelial cells (BECs) (Fig. 1b). In mice, acute or chronic cigarette smoke exposure increased pulmonary IL-33 expression, viral infection with IAV or RSV but not fungal infection with Alternaria induced its release, and administration of rIL-33 exacerbated virus-induced inflammation. These studies also showed that acute smoke exposure reduced IL-5 and IL-13 responses to $\mathrm{rLL}-33$, as well as the numbers of IL-13-expressing ILCS and their production of IL-5 and IL-13 in response to IL-33. Smoke also suppressed ST2 expression on ILC2s whilst increasing expression on macrophages and NK cells, and reduced cytokine production by ILC2 and NK cells. The absence of IL-33, in IL-33 or ST2-deficient mice or treatment with an ST2 inhibitor, suppressed IAV-induced exacerbation of acute cigarette smoke-driven inflammation. ILC2-deficiency also protected against CS-induced emphysema in mice. ${ }^{158}$ Mechanistically IL-33 enhanced macrophage and NK responses to IAV following acute smoke exposure in wild-type but not ST2-deficient mice, which were supported by ex vivo mouse studies. Thus, smoke exposure increases IL-33 expression and infection induces its release amplifying type- 1 inflammatory responses by promoting macrophage and NK cell function. More generally, smoke alters lung immunity to facilitate IL-33 exaggeration of pro-inflammatory responses to infection that exacerbates the underlying disease. ${ }^{14}$ The roles of ILC2s in the pathogenesis of COPD and infection rather than in acute smoke exposure remain to be defined. These roles could be elucidated using mouse models that recapitulate the hallmark features of the human disease as well as human tissues and cells. ${ }^{159-161}$

In support of the generalizability of these observations to chronic respiratory diseases, IPF patients have increased IL-25 expression and ILC2s in their BALF, and IL-33 is constitutively or inducibly expressed in lung BECs and macrophages, respectively, in bleomycin-induced experimental pulmonary fibrosis. ${ }^{162}$ Experimentally, Schistosoma mansonii-induced granulomas and fibrosis were dependent on IL-25 and IL-17, and IL-25-induced fibrosis required ILC2s in wild-type compared to ILC2-deficient (Rora ${ }^{s g / s g}$ ) mice. ${ }^{163}$ They extended these findings by showing that ILC2s regulate fibrosis in anti-CD90.2 treated T-cell and B-cell-deficient mice, and that IL-13 ${ }^{+}$ILC2s regulate collagen deposition in adoptive transfer studies in $1113^{-1-}$ mice. Others showed that combined targeting of TSLP, IL-25 and IL-33 suppressed type-2driven inflammatory and IL-13 producing ILC2 responses, airway remodeling and fibrosis during $S$. mansonii infection and HDMinduced allergic lung inflammation. ${ }^{164}$ Inhibition of IL-33 activity or depletion of alveolar macrophages decreased, whereas treatment with rlL-33 or adoptive transfer of ILC2s increased, inflammation and fibrosis. Indeed, IL-33 induced M2 macrophage polarization and expansion of ILC2s with pro-inflammatory and pro-fibrotic responses in vitro and in vivo. Thus, IL-33 is proinflammatory and pro-fibrotic and initiates and progresses pulmonary fibrosis involving macrophages and ILC2s.

Other recent studies found elevated levels of IL-9 in airways of cystic fibrosis patients, and in mice that IL-9 induces IL-2 production by mast cells that promotes IL- $25^{+}$ILC2 and Th9 cell proliferation. ${ }^{165}$ Blocking IL-9 or c-Kit (CD117) prevented these effects. Thus, targeting IL-9 may reduce Th9 and ILC2-associated lung inflammation in fibrotic lung diseases. 
ILC2s in respiratory infections

ILC2s have been implicated in both protection against and pathogenesis of parasitic, viral and bacterial infections and their exacerbations of chronic respiratory diseases (Fig. 1). Their involvement in parasitic infections has been extensively reviewed elsewhere. ${ }^{166}$ Therefore, we focus on role of ILC2s in respiratory viral infections.

Rhinovirus

Recent studies have made substantial advances in understanding the role of ILC2s in RV infections in early life, which induce mucus hypersecretion and AHR. ${ }^{167}$ Profiling of responses of neonatal ( 6 day-old) or adult ( 8 week-old) mice to RV infection over 28 days showed that neonatal mice had increased IL-13 production from ILC2s, but reduced IFN- $\gamma$, IL-12 and TNF- $a$ expression compared to adults. ${ }^{167}$ IL-25 attenuated increases in ILC2s, mucus hypersecretion and AHR. They then showed that intranasal administration of recombinant ( $r$ )IFN- $\gamma$ protein or Rora inhibitors reduced these RVinduced IL-13 and ILC2 responses and mucus hypersecretion. ${ }^{168,169}$ Treatment of lung ILC2s ex vivo with $r$ IFN- $\gamma$ reduced IL-5, IL-13, IL-17RB, ST2, and GATA3 expression, or with Rora inhibitor blocked the expansion of IL-25-induced or IL-33-induced ILC2s and IL-13 release. ${ }^{169,170}$ Infected Rora ${ }^{\mathrm{sg} / \mathrm{sg}}$ mice had reduced expansion of ILC2s, and sorted ILC2s induced an asthma-like phenotype in naïve young or adult mice. ${ }^{169}$ Most recently IL-33 and TSLP were shown to be induced in airway epithelial cells by neonatal infection and were required for maximal IL-25 expression, ILC2 development, mucus hypersecretion and AHR. ${ }^{170}$ Thus, early-life RV infection induces an interplay of IL-25, IL-33 and TSLPdriven type- 2 and ILC2 responses that require Rora and contribute to mucus hypersecretion, AHR and potentially asthma. In contrast, IFN- $\gamma$ inhibits ILC2 expansion, IL-13 expression and mucus hypersecretion.

Translational studies have been performed in experimental RVinduced asthma exacerbations in human adults. ${ }^{171}$ Infection increased IL-4, IL-5, IL-13 and IL-33 in asthmatic airways that correlated with disease severity compared to healthy controls. IL33 levels also correlated with IL-5 and IL-13 levels. In vitro RV infection of BECs induced IL-33 and culture of pBECs, peripheral Tcells or ILC2s with supernatants from RV-infected BECs induced IL33-dependent Th2 cytokine release without affecting IL-25, IL-33 or TSLP. This shows that RV-induced asthma exacerbations in adults involve the induction of type-2 cytokines including IL-33, and that T-cells and ILC2s are mechanistic links. Thus, IL-33 may be a therapeutic target in asthma exacerbations.

\section{Respiratory syncytial virus}

Similar observations have been made with RSV strengthening generalizability. IL-33-induced increases in ILC2s play critical roles in the pathogenesis of RSV infection only in early life. Increased levels of IL-33 and IL-13 were detected in nasal secretions of hospitalized infants with RSV that subsided during recovery. ${ }^{116}$ In mice RSV infection in neonates but not adults induced rapid increases in IL-33 expression and ILC2s in the lungs. Suppression of IL-33 responses inhibited RSV-induced Th2 inflammation airway eosinophilia, mucus hypersecretion and AHR with opposite effects induced by rlL-33 administration. Others investigated the mechanisms involved and showed that RSV infection in wild-type mice increased IL-13, IL-33 and TSLP levels and the numbers of IL-13 producing ILC2s. ${ }^{172}$ Deletion of TSLP reduced IL-13 levels, IL-13-producing ILC2s, mucus hypersecretion, AHR and weight loss without affecting viral load. They also showed that RSV-induced STAT1 responses were required for the control of immunopathologic IL-5 $5^{+}$and IL-13 $3^{+}$ ILC2s. Both intrinsic and extrinsic factors caused this dysfunction with extrinsic IL-33 promoting ILC2s. These studies show that IL33 and TSLP are required to induce $\mathrm{IL}-13^{+} \mathrm{ILC} 2 \mathrm{~s}$ and Th2associated disease during neonatal RSV infection, and that
STAT1 opposes these effects. Thus, again IL-33 and TSLP may be therapeutic targets.

Influenza $A$ virus

IAV infection of mice leads to the accumulation of ILC2s in the lungs, and their depletion during infection caused the loss of epithelial integrity and impaired lung function and airway remodeling. ${ }^{5}$ These events could be reversed by ILC2-derived amphiregulin. Respiratory viral infections typically induce type-1 pro-inflammatory responses though type-2 responses also arise in a tissue protective role and may lead to asthma exacerbations. Infection with pandemic strains of IAV induces IFN- $\gamma$ responses that restricts protective ILC2 function. A recent study showed that genetic IFN- $\gamma$ deficiency or anti-IFN- $\gamma$ treatment during IAV infection did not increase ILC2s but enhanced their activity and release of IL-5 and amphiregulin and improved tissue protection without affecting viral load or clearance. ${ }^{173}$ These effects were dependent on IL-5 and were not observed in ILC2-deficient mice.

Asthma and COPD patients are more susceptible to IAV infections that exacerbate the underlying disease and ILC2s may be involved. However, the mechanisms involved are poorly understood. IAV infection induced AHR independently of T- and B-cells in Rag2 ${ }^{-1-}$ mice but did require ST2. ${ }^{174}$ Infection lead to IL33 production in alveolar macrophages and increased the numbers of ILCs, which did not occur in $1113^{-1-}$ or Rag $2^{-/-}$mice or with anti-CD90.2 treatment. Others showed that IAV infections in mice also induced robust but transient IL-5 production from infiltrating $\mathrm{c}-\mathrm{kit}^{+} \mathrm{ST}_{2}^{+}$ILC2s and concomitant eosinophil influx into the airways, particularly during recovery. ${ }^{175}$ The effects were abrogated with anti-CD90.2 treatment. In these studies, NKT cells and alveolar macrophages were the sources of IL-33. A follow up study found increased ILC1s in COPD patients that were associated with disease severity and exacerbation susceptibility. ${ }^{14}$ Experimentally they showed that ILC2s can also be plastic during IAV infection. They developed ILC1 characteristics with reduced GATA-3 expression and produced IFN- $\gamma, \mathrm{IL}-12$ and IL-18. These ILC1s reinforced T-bet dependent virus-induced inflammation. Translating their findings, they showed that IL-12 converted human ILC2s into ILC1s.

Thus, ILC2s are crucial in restoring the airway epithelium after IAV infection, but IFN- $\gamma$ restricts their function promoting pathogenesis. Consequently, increasing ILC2 activity is a potential therapeutic strategy. In exacerbations of chronic respiratory diseases IAV-induces AHR through an IL-13/-33/ILC2 axis, and interactions between ILC2s and IL-33 producing NKT cells and alveolar macrophages leads to high levels of IL-5 production by ILC2s and eosinophilopoiesis during recovery from IAV infection that may exacerbate asthma. In contrast, ILC2 plasticity toward ILC1s exacerbates virus-induced inflammation that may have adverse consequences in COPD. Thus, early IL-13 or IL-33 and/or later anti-IL-5 treatment may be beneficial in asthma but maintaining ILC2 function may be protective in COPD.

\section{CONCLUSION}

Despite the recent explosion of studies, particularly using 'omics technologies, investigating the biology of ILC2s in multiple contexts, namely in the respiratory tract, there are still many unanswered questions. These include, a consensus for consistency in identifying and reporting of ILC2s to limit issues with reproducibility in the identification and isolation of ILC2s in both mice and humans, clarifying their roles in different disease states, and defining the potential strategies for therapeutic manipulation. There are emerging anti-inflammatory roles for ILC2s that interact with Tregs that need to be defined. Numerous influencers of ILC2 function are being identified including novel roles for circadian rhythm, sex hormones, age, and neurotransmitters. ILC2s have 
important roles in chronic lung diseases such as asthma, COPD and infection that are only just beginning to be unraveled. They may promote the development of asthma and be involved in pulmonary fibrosis, but their numbers and function are reduced in COPD. ILC2s may also have pathogenic roles in RV and RSV infections, particularly in early life, but may be critical for tissue restoration after IAV infection. Their profiles and roles in disease states may be further defined using single cell sequencing. ILC2s may both modulate and be modulated by microbiota in the lung and gut and have local and systemic effects that differentially affect chronic respiratory diseases and infections. This may be dependent on host genetics and consequently susceptibility to infection. Fully elucidating the roles of ILC2s in development and disease and the generation of new ways to specifically modulate them has the potential to substantially impact the ways that these issues are prevented and treated.

\section{ACKNOWLEDGEMENTS}

The authors apologize in advance for and regret any omissions, either unintended or due to space limitations. We acknowledge Guy Cameron and Svenja Loering for their assistance with preparing figures and tables. M.R.S. is supported by an Australian Research Council Discovery Early Career Researcher Award, G.T.B. and P.M.H. are supported by fellowships from the National Health and Medical Research Council (Australia). P.M.H. is also supported by a fellowship from the Faculty of Health, University of Newcastle.

\section{ADDITIONAL INFORMATION}

Competing interests: The authors declare no competing interests.

Publisher's note: Springer Nature remains neutral with regard to jurisdictional claims in published maps and institutional affiliations.

\section{REFERENCES}

1. Neill, D. R. et al. Nuocytes represent a new innate effector leukocyte that mediates type-2 immunity. Nature 464, 1367-1370 (2010).

2. Halim, T. Y. et al. Group 2 innate lymphoid cells are critical for the initiation of adaptive $\mathrm{T}$ helper 2 cell-mediated allergic lung inflammation. Immunity $\mathbf{4 0}$, 425-435 (2014).

3. Spits, H. et al. Innate lymphoid cells--a proposal for uniform nomenclature. Nat. Rev. Immunol. 13, 145-149 (2013).

4. Bar-Ephraim, Y. E. \& Mebius, R. E. Innate lymphoid cells in secondary lymphoid organs. Immunol. Rev. 271, 185-199 (2016).

5. Monticelli, L. A. et al. Innate lymphoid cells promote lung-tissue homeostasis after infection with influenza virus. Nat. Immunol. 12, 1045-1054 (2011).

6. Sonnenberg, G. F., Fouser, L. A. \& Artis, D. Border patrol: regulation of immunity, inflammation and tissue homeostasis at barrier surfaces by IL-22. Nat. Immunol. 12, 383-390 (2011)

7. Li, Z. et al. Epidermal Notch1 recruits RORgamma(+) group 3 innate lymphoid cells to orchestrate normal skin repair. Nat. Commun. 7, 11394 (2016).

8. Lee, M. W. et al. Activated type 2 innate lymphoid cells regulate beige fat biogenesis. Cell 160, 74-87 (2015).

9. Sanos, S. L., Vonarbourg, C., Mortha, A. \& Diefenbach, A. Control of epithelial cell function by interleukin-22-producing RORgammat+innate lymphoid cells. Immunology 132, 453-465 (2011).

10. Sawa, S. et al. RORgammat+innate lymphoid cells regulate intestinal homeostasis by integrating negative signals from the symbiotic microbiota. Nat. Immunol. 12, 320-326 (2011).

11. Sonnenberg, G. F. et al. Innate lymphoid cells promote anatomical containment of lymphoid-resident commensal bacteria. Science 336, 1321-1325 (2012).

12. Gladiator, A., Wangler, N., Trautwein-Weidner, K. \& LeibundGut-Landmann, S. Cutting edge: IL-17-secreting innate lymphoid cells are essential for host defense against fungal infection. J. Immunol. 190, 521-525 (2013).

13. Bal, S. M. et al. IL-1beta, IL- 4 and IL-12 control the fate of group 2 innate lymphoid cells in human airway inflammation in the lungs. Nat. Immunol. 17, 636-645 (2016).

14. Silver, J. S. et al. Inflammatory triggers associated with exacerbations of COPD orchestrate plasticity of group 2 innate lymphoid cells in the lungs. Nat. Immunol. 17, 626-635 (2016).
15. Walker, J. A. \& McKenzie, A. N. J. TH2 cell development and function. Nat. Rev. Immunol. 18, 121-133 (2018).

16. Mattner, J. \& Wirtz, S. Friend or Foe? The ambiguous role of innate lymphoid cells in cancer development. Trends Immunol. 38, 29-38 (2017).

17. Huang, Y. et al. IL-25-responsive, lineage-negative KLRG1(hi) cells are multipotential 'inflammatory' type 2 innate lymphoid cells. Nat. Immunol. 16, 161-169 (2015).

18. Huang, Y. et al. S1P-dependent interorgan trafficking of group 2 innate lymphoid cells supports host defense. Science (New York, NY) 359, 114-119 (2018).

19. Huang, Y. \& Paul, W. E. Inflammatory group 2 innate lymphoid cells. Int. Immunol. 28, 23-28 (2016).

20. Hoyler, T. et al. The transcription factor GATA-3 controls cell fate and maintenance of type 2 innate lymphoid cells. Immunity 37, 634-648 (2012).

21. Mjosberg, J. et al. The transcription factor GATA3 is essential for the function of human type 2 innate lymphoid cells. Immunity 37, 649-659 (2012).

22. Li, B. W. S. et al. Group 2 innate lymphoid cells exhibit a dynamic phenotype in allergic airway inflammation. Front. Immunol. 8, 1684 (2017).

23. Klein Wolterink, R. G. et al. Essential, dose-dependent role for the transcription factor Gata3 in the development of IL-5+and IL-13+type 2 innate lymphoid cells. Proc. Natl Acad. Sci. USA 110, 10240-10245 (2013).

24. Wong, S. H. et al. Transcription factor ROR alpha is critical for nuocyte development. Nat. Immunol. 13, 229-236 (2012).

25. Gentek, R. et al. Modulation of signal strength switches notch from an inducer of T cells to an inducer of ILC2. Front. Immunol. 4, 334 (2013).

26. Seillet, C. et al. Nfil3 is required for the development of all innate lymphoid cell subsets. J. Exp. Med. 211, 1733-1740 (2014).

27. Geiger, T. L. et al. Nfil3 is crucial for development of innate lymphoid cells and host protection against intestinal pathogens. J. Exp. Med. 211, 1723-1731 (2014).

28. Mielke, L. A. et al. TCF-1 controls ILC2 and NKp46+RORgammat+innate lymphocyte differentiation and protection in intestinal inflammation. J. Immunol. 191, 4383-4391 (2013).

29. Yang, Q. et al. T cell factor 1 is required for group 2 innate lymphoid cell generation. Immunity 38, 694-704 (2013).

30. Spooner, C. J. et al. Specification of type 2 innate lymphocytes by the transcriptional determinant Gfi1. Nat. Immunol. 14, 1229-1236 (2013).

31. Wang, H. C. et al. Downregulation of E protein activity augments an ILC2 differentiation program in the thymus. J. Immunol. (Baltim., Md: 1950) 198, 3149-3156 (2017).

32. Walker, J. A. \& McKenzie, A. N. Development and function of group 2 innate lymphoid cells. Curr. Opin. Immunol. 25, 148-155 (2013).

33. Stier, M. T. et al. IL-33 promotes the egress of group 2 innate lymphoid cells from the bone marrow. J. Exp. Med. 215, 263-281 (2018).

34. Miyazaki, M. et al. The E-Id protein axis specifies adaptive lymphoid cell identity and suppresses thymic innate lymphoid cell development. Immunity 46, 818-834.e814 (2017).

35. Seillet, $C$. et al. Deciphering the innate lymphoid cell transcriptional program. Cell Rep. 17, 436-447 (2016).

36. Yu, Y. et al. Single-cell RNA-seq identifies a PD-1(hi) ILC progenitor and defines its development pathway. Nature 539, 102-106 (2016).

37. Taylor, S. et al. PD-1 regulates KLRG1(+) group 2 innate lymphoid cells. J. Exp. Med. 214, 1663-1678 (2017).

38. Schwartz, C. et al. ILC2s regulate adaptive Th2 cell functions via PD-L1 checkpoint control. J. Exp. Med. 214, 2507-2521 (2017).

39. Starkey, M. R. et al. Programmed death ligand 1 promotes early-life chlamydia respiratory infection-induced severe allergic airway disease. Am. J. Respir. Cell Mol. Biol. 54, 493-503 (2016).

40. Starkey, M. R. et al. Constitutive production of IL-13 promotes early-life Chlamydia respiratory infection and allergic airway disease. Mucosal Immunol. 6, 569-579 (2013).

41. Gollwitzer, E. S. et al. Lung microbiota promotes tolerance to allergens in neonates via PD-L1. Nat. Med. 20, 642-647 (2014).

42. Gury-BenAri, M. et al. The spectrum and regulatory landscape of intestinal innate lymphoid cells are shaped by the microbiome. Cell 166, 1231-1246. e1213 (2016).

43. Lim, A. I. et al. Systemic human ILC precursors provide a substrate for tissue ILC differentiation. Cell 168, 1086-1100.e1010 (2017).

44. Robinette, M. L. et al. Transcriptional programs define molecular characteristics of innate lymphoid cell classes and subsets. Nat. Immunol. 16, 306-317 (2015).

45. Chea, S. et al. Single-cell gene expression analyses reveal heterogeneous responsiveness of fetal innate lymphoid progenitors to notch signaling. Cell Rep. 14, 1500-1516 (2016).

46. Shih, H. Y. et al. Developmental acquisition of regulomes underlies innate lymphoid cell functionality. Cell 165, 1120-1133 (2016). 
47. Koues, O. I. et al. Distinct gene regulatory pathways for human innate versus adaptive lymphoid cells. Cell 165, 1134-1146 (2016).

48. Bjorklund, A. K. et al. The heterogeneity of human CD127(+) innate lymphoid cells revealed by single-cell RNA sequencing. Nat. Immunol. 17, 451-460 (2016).

49. Vivier, E. et al. Innate lymphoid cells: 10 years on. Cell 174, 1054-1066 (2018).

50. Krabbendam, L., Bal, S. M., Spits, H. \& Golebski, K. New insights into the function, development, and plasticity of type 2 innate lymphoid cells. Immunol. Rev. 286, 74-85 (2018).

51. Bernink, J. H. et al. Interleukin-12 and -23 control plasticity of CD127(+) Group 1 and Group 3 innate lymphoid cells in the intestinal lamina propria. Immunity 43, 146-160 (2015)

52. Cella, M., Otero, K. \& Colonna, M. Expansion of human NK-22 cells with IL-7, IL-2, and IL-1beta reveals intrinsic functional plasticity. Proc. Natl Acad. Sci. USA 107 10961-10966 (2010)

53. Okuda, Y. et al. [A Retrospective Investigation of Lacrimation in Patients Treated with S-1]. Gan. To. Kagaku. Ryoho. 45, 265-268 (2018).

54. Vonarbourg, C. et al. Regulated expression of nuclear receptor RORgammat confers distinct functional fates to NK cell receptor-expressing RORgammat(+) innate lymphocytes. Immunity 33, 736-751 (2010).

55. Klose, C. S. et al. Differentiation of type 1 ILCs from a common progenitor to all helper-like innate lymphoid cell lineages. Cell 157, 340-356 (2014).

56. Rankin, L. C. et al. The transcription factor T-bet is essential for the development of NKp46(+) innate lymphocytes via the Notch pathway. Nat. Immunol. 14, 389-395 (2013).

57. Sciume, G. et al. Distinct requirements for T-bet in gut innate lymphoid cells. J. Exp. Med. 209, 2331-2338 (2012).

58. Viant, C. et al. Transforming growth factor-beta and Notch ligands act as opposing environmental cues in regulating the plasticity of type 3 ILCs. Sci. Signal. 9, ra46 (2016).

59. Lee, J. S. et al. AHR drives the development of gut ILC22 cells and postnatal lymphoid tissues via pathways dependent on and independent of Notch. Nat. Immunol. 13, 144-151 (2011).

60. Zhang, K. et al. Notch signaling promotes the plasticity of Group-2 innate lymphoid cells. J. Immunol. 198, 1798-1803 (2018).

61. Ohne, Y. et al. IL-1 is a critical regulator of group 2 innate lymphoid cell function and plasticity. Nat. Immunol. 17, 646-655 (2016).

62. Lim, A. I. et al. IL-12 drives functional plasticity of human group 2 innate lymphoid cells. J. Exp. Med. 213, 569-583 (2016).

63. Ricardo-Gonzalez, R. R. et al. Tissue signals imprint ILC2 identity with anticipatory function. Nat. Immunol. 19, 1093-1099 (2018).

64. Freud, A. G. \& Caligiuri, M. A. Human natural killer cell development. Immunol. Rev. 214, 56-72 (2006).

65. Simoni, Y. et al. Human innate lymphoid cell subsets possess tissue-type based heterogeneity in phenotype and frequency. Immunity 46, 148-161 (2017).

66. Scoville, S. D. et al. A progenitor cell expressing transcription factor rorgammat generates all human innate lymphoid cell subsets. Immunity 44, 1140-1150 (2016).

67. Serafini, N. et al. Gata3 drives development of RORgammat+group 3 innate lymphoid cells. J. Exp. Med. 211, 199-208 (2014).

68. Yagi, R. et al. The transcription factor GATA3 is critical for the development of all IL-7Ralpha-expressing innate lymphoid cells. Immunity 40, 378-388 (2014).

69. Rauber, S. et al. Resolution of inflammation by interleukin-9-producing type 2 innate lymphoid cells. Nat. Med. 23, 938-944 (2017).

70. Lai, D. et al. Group 2 innate lymphoid cells protect lung endothelial cells from pyroptosis in sepsis. Cell death \& Dis. 9, 369 (2018).

71. Omata, Y. et al. Group 2 innate lymphoid cells attenuate inflammatory arthritis and protect from bone destruction in mice. Cell Rep. 24, 169-180 (2018).

72. Mohapatra, A. et al. Group 2 innate lymphoid cells utilize the IRF4-IL-9 module to coordinate epithelial cell maintenance of lung homeostasis. Mucosal Immunol. 9, 275-286 (2016)

73. Gasteiger, G., Fan, X., Dikiy, S., Lee, S. Y. \& Rudensky, A. Y. Tissue residency of innate lymphoid cells in lymphoid and nonlymphoid organs. Science (New York, NY) 350, 981-985 (2015).

74. Maazi, H. et al. ICOS:ICOS-ligand interaction is required for type 2 innate lymphoid cell function, homeostasis, and induction of airway hyperreactivity. Immunity 42, 538-551 (2015).

75. Molofsky, A. B. et al. Interleukin-33 and interferon-gamma counter-regulate Group 2 innate lymphoid cell activation during immune perturbation. Immunity 43, 161-174 (2015).

76. Rigas, D. et al. Type 2 innate lymphoid cell suppression by regulatory T cells attenuates airway hyperreactivity and requires inducible T-cell costimulatorinducible T-cell costimulator ligand interaction. J. Allergy Clin. Immunol. 139, 1468-1477.e1462 (2017).
77. Ogasawara, N. et al. IL-10, TGF-beta, and glucocorticoid prevent the production of type 2 cytokines in human group 2 innate lymphoid cells. J. Allergy Clin. Immunol. 141, 1147-1151.e1148 (2018).

78. Denney, L. et al. Pulmonary epithelial cell-derived cytokine TGF-beta1 is a critical cofactor for enhanced innate lymphoid cell function. Immunity 43, 945-958 (2015).

79. Halim, T. Y. F. et al. Tissue-restricted adaptive Type 2 immunity is orchestrated by expression of the costimulatory molecule OX40L on Group 2 innate lymphoid cells. Immunity 48, 1195-1207.e1196 (2018).

80. Scheiermann C., Gibbs J., Ince L., Loudon A. Clocking in to immunity. Nat. Rev. Immunol. 18, 423-437 (2018).

81. Stratmann, M. \& Schibler, U. Properties, entrainment, and physiological functions of mammalian peripheral oscillators. J. Biol. Rhythms 21, 494-506 (2006).

82. Mohawk, J. A., Green, C. B. \& Takahashi, J. S. Central and peripheral circadian clocks in mammals. Annu. Rev. Neurosci. 35, 445-462 (2012).

83. Sato, T. K. et al. A functional genomics strategy reveals Rora as a component of the mammalian circadian clock. Neuron 43, 527-537 (2004).

84. $\mathrm{Yu}, \mathrm{X}$. et al. TH17 cell differentiation is regulated by the circadian clock. Science (New York, NY) 342, 727-730 (2013).

85. Gibbs, J. E. et al. The nuclear receptor REV-ERBalpha mediates circadian regulation of innate immunity through selective regulation of inflammatory cytokines. Proc. . Natl. Acad. Sci. USA 109, 582-587 (2012).

86. Pariollaud M. et al. Circadian clock component REV-ERBalpha controls homeostatic regulation of pulmonary inflammation. J. Clin. Invest. 128, 2281-2296 (2018).

87. Nguyen, K. D. et al. Circadian gene Bmal1 regulates diurnal oscillations of Ly6C (hi) inflammatory monocytes. Science (New York, NY) 341, 1483-1488 (2013).

88. Curtis, A. M. et al. Circadian control of innate immunity in macrophages by miR155 targeting Bmal1. Proc. Natl. Acad. Sci. USA 112, 7231-7236 (2015).

89. Singh, P. B. et al. MicroRNA regulation of type 2 innate lymphoid cell homeostasis and function in allergic inflammation. J. Exp. Med. 214, 3627-3643 (2017).

90. Johansson, K., Malmhall, C., Ramos-Ramirez, P. \& Radinger, M. MicroRNA-155 is a critical regulator of type 2 innate lymphoid cells and IL-33 signaling in experimental models of allergic airway inflammation. J. Allergy Clin. Immunol. 139, 1007-1016.e1009 (2017).

91. Harmar, A. J. et al. The VPAC(2) receptor is essential for circadian function in the mouse suprachiasmatic nuclei. Cell 109, 497-508 (2002).

92. Nussbaum, J. C. et al. Type 2 innate lymphoid cells control eosinophil homeostasis. Nature 502, 245-248 (2013).

93. Carey, M. A. et al. It's all about sex: gender, lung development and lung disease. Trends Endocrinol. Metab.: TEM 18, 308-313 (2007).

94. Almqvist, C., Worm, M. \& Leynaert, B. working group of GALENWPG. Impact of gender on asthma in childhood and adolescence: a GA2LEN review. Allergy $\mathbf{6 3}$, 47-57 (2008).

95. Cephus, J. Y. et al. Testosterone Attenuates Group 2 Innate Lymphoid CellMediated Airwaylnflammation. Cell Rep. 21, 2487-2499 (2017).

96. Oertelt-Prigione, $\mathrm{S}$. The influence of sex and gender on the immune response. Autoimmun. Rev. 11, A479-A485 (2012).

97. Kawana, K., Kawana, Y. \& Schust, D. J. Female steroid hormones use signal transducers and activators of transcription protein-mediated pathways to modulate the expression of T-bet in epithelial cells: a mechanism for local immune regulation in the human reproductive tract. Mol. Endocrinol. (Baltim., Md) 19, 2047-2059 (2005)

98. Pan, T. et al. 17beta-Oestradiol enhances the expansion and activation of myeloid-derived suppressor cells via signal transducer and activator of transcription (STAT)-3 signalling in human pregnancy. Clin. Exp. Immunol. 185, 86-97 (2016).

99. Douin-Echinard, V. et al. Estrogen receptor alpha, but not beta, is required for optimal dendritic cell differentiation and [corrected] CD40-induced cytokine production. J. Immunol. (Baltim., Md: 1950) 180, 3661-3669 (2008).

100. Laffont, S., Seillet, C. \& Guery, J. C. Estrogen Receptor-Dependent Regulation of Dendritic Cell Development and Function. Front. Immunol. 8, 108 (2017).

101. Butts, C. L. et al. Progesterone inhibits mature rat dendritic cells in a receptormediated fashion. Int. Immunol. 19, 287-296 (2007).

102. Zhao, X. et al. Progesterone enhances immunoregulatory activity of human mesenchymal stem cells via PGE2 and IL-6. Am. J. Reprod. Immunol. (New York, NY: 1989) 68, 290-300 (2012).

103. Enninga, E. A. et al. Immunomodulatory effects of sex hormones: requirements for pregnancy and relevance in melanoma. Mayo Clin. Proc. 89 520-535 (2014).

104. Hepworth, M. R., Hardman, M. J. \& Grencis, R. K. The role of sex hormones in the development of Th2 immunity in a gender-biased model of Trichuris muris infection. Eur. J. Immunol. 40, 406-416 (2010). 
105. Arruvito, L. et al. NK cells expressing a progesterone receptor are susceptible to progesterone-induced apoptosis. J. Immunol. (Baltim., Md: 1950) 180, 5746-5753 (2008).

106. Llewelyn, D. E., Read, G. F. \& Hillier, S. G. Proceedings: Novel procedure for the simultaneous determination of testosterone and 5alpha-dihydrotestosterone concentrations in unpurified plasma extracts by radioimmunoassay. J. Endocrinol. 67, 7p-8p (1975).

107. Penning, T. M., Bauman, D. R., Jin, Y. \& Rizner, T. L. Identification of the molecular switch that regulates access of 5alpha-DHT to the androgen receptor. Mol. Cell. Endocrinol. 265-266, 77-82 (2007).

108. Laffont, $\mathrm{S}$. et al. Androgen signaling negatively controls group 2 innate lymphoid cells. J. Exp. Med. 214, 1581-1592 (2017).

109. Kadel, S. et al. A Major Population of Functional KLRG1(-) ILC2s in Female Lungs Contributes to a Sex Bias in ILC2 Numbers. ImmunoHorizons 2, 74-86 (2018).

110. Russi, A. E., Ebel, M. E., Yang, Y. \& Brown, M. A. Male-specific IL-33 expression regulates sex-dimorphic EAE susceptibility. Proc. Natl Acad. Sci. USA 115, E1520-e1529 (2018).

111. Russi, A. E., Walker-Caulfield, M. E., Ebel, M. E. \& Brown, M. A. Cutting edge: c-Kit signaling differentially regulates type 2 innate lymphoid cell accumulation and susceptibility to central nervous system demyelination in male and female SJL mice. J. Immunol. (Baltim., Md: 1950) 194, 5609-5613 (2015).

112. Bartemes, K., Chen, C. C., lijima, K., Drake, L. \& Kita, H. IL-33-Responsive Group 2 Innate Lymphoid Cells Are Regulated by Female Sex Hormones in the Uterus. J. Immunol. (Baltim., Md: 1950) 200, 229-236 (2018).

113. Saluzzo, S. et al. First-Breath-Induced Type 2 Pathways Shape the Lung ImmuneEnvironment. . Cell Rep. 18, 1893-1905 (2017).

114. Steer, C. A. et al. Group 2 innate lymphoid cell activation in the neonatal lung drives type 2 immunity and allergen sensitization. J. Allergy Clin. Immunol. 140, 593-595.e593 (2017).

115. de Kleer, I. M. et al. Perinatal Activation of the Interleukin-33 Pathway Promotes Type 2 Immunity in the Developing Lung. Immunity 45, 1285-1298 (2016).

116. Saravia, J. et al. Respiratory Syncytial Virus Disease Is Mediated by Age-Variable IL-33. PLoS. Pathog. 11, e1005217 (2015).

117. PrabhuDas, M. et al. Challenges in infant immunity: implications for responses to infection and vaccines. Nat. Immunol. 12, 189-194 (2011).

118. Mohr, E. \& Siegrist, C. A. Vaccination in early life: standing up to the challenges. Curr. Opin. Immunol. 41, 1-8 (2016).

119. Kurowska-Stolarska, M. et al. IL-33 amplifies the polarization of alternatively activated macrophages that contribute to airway inflammation. J. Immunol. (Baltim., Md: 1950) 183, 6469-6477 (2009).

120. Jones, C. V. et al. M2 macrophage polarisation is associated with alveolar formation during postnatal lung development. Respir. Res. 14, 41 (2013).

121. Lechner, A. J. et al. Recruited Monocytes and Type 2 Immunity Promote Lung Regeneration following Pneumonectomy. Cell. Stem. Cell. 21, 120-134.e127 (2017)

122. Sugita, K. et al. Type 2 innate lymphoid cells disrupt bronchial epithelial barrier integrity by targeting tight junctions through IL-13 in asthmatic patients. J. Allergy Clin. Immunol. 141, 300-310.e311 (2018).

123. Franco, R., Schoneveld, O. J., Pappa, A. \& Panayiotidis, M. I. The central role of glutathione in the pathophysiology of human diseases. Arch. Physiol. Biochem. 113, 234-258 (2007).

124. Papa, I. et al. TFH-derived dopamine accelerates productive synapses in germinal centres. Nature 547, 318-323 (2017).

125. Gadani, S. P., Smirnov, I., Smith, A. T., Overall, C. C. \& Kipnis, J. Characterization of meningeal type 2 innate lymphocytes and their response to CNS injury. J. Exp. Med. 214, 285-296 (2017).

126. Hedrick, J. A. et al. Identification of a human gastrointestinal tract and immune system receptor for the peptide neuromedin U. Mol. Pharmacol. 58, 870-875 (2000).

127. Moriyama, M. et al. The neuropeptide neuromedin U promotes inflammation by direct activation of mast cells. J. Exp. Med. 202, 217-224 (2005).

128. Moriyama, $M$. et al. The neuropeptide neuromedin $\mathrm{U}$ activates eosinophils and is involved in allergen-induced eosinophilia. Am. J. Physiol. Lung Cell. Mol. Physiol. 290, L971-L977 (2006).

129. Klose, C. S. N. et al. The neuropeptide neuromedin U stimulates innate lymphoid cells and type 2 inflammation. Nature 549, 282-286 (2017).

130. Wallrapp, A. et al. The neuropeptide NMU amplifies ILC2-driven allergic lung inflammation. Nature 549, 351-356 (2017).

131. Cardoso, V. et al. Neuronal regulation of type 2 innate lymphoid cells via neuromedin U. Nature 549, 277-281 (2017).

132. Modena, B. D. et al. Gene Expression Correlated with Severe Asthma Characteristics Reveals Heterogeneous Mechanisms of Severe Disease. Am. J. Respir. Crit. Care. Med. 195, 1449-1463 (2017).
133. Beale, J. et al. Rhinovirus-induced IL-25 in asthma exacerbation drives type 2 immunity and allergic pulmonary inflammation. Sci. Transl. Med. 6, 256ra134 (2014).

134. Sui $\mathrm{P}$. et al. Pulmonary neuroendocrine cells amplify allergic asthma responses. Science (New York, NY) 360 (2018)

135. Moriyama, S. et al. beta2-adrenergic receptor-mediated negative regulation of group 2 innate lymphoid cell responses. Science (New York, NY) 359, 1056-1061 (2018).

136. Hammad, H. \& Lambrecht, B. N. Barrier Epithelial Cells and the Control of Type 2 Immunity. Immunity 43, 29-40 (2015).

137. Kubo, M. Innate and adaptive type 2 immunity in lung allergic inflammation. Immunol. Rev. 278, 162-172 (2017)

138. Hansbro, P. M., Beagley, K. W., Horvat, J. C. \& Gibson, P. G. Role of atypical bacterial infection of the lung in predisposition/protection of asthma. Pharmacol. Ther. 101, 193-210 (2004).

139. Hansbro, N. G., Horvat, J. C., Wark, P. A. \& Hansbro, P. M. Understanding the mechanisms of viral induced asthma: new therapeutic directions. Pharmacol. Ther. 117, 313-353 (2008).

140. Hansbro, P. M. et al. Mechanisms and treatments for severe, steroid-resistant allergic airway disease and asthma. Immunol. Rev. 278, 41-62 (2017).

141. Hansbro, P. M., Kaiko, G. E. \& Foster, P. S. Cytokine/anti-cytokine therapy - novel treatments for asthma? Br. J. Pharmacol. 163, 81-95 (2011).

142. Kim, R. Y. et al. MicroRNA-21 drives severe, steroid-insensitive experimental asthma by amplifying phosphoinositide 3-kinase-mediated suppression of histone deacetylase 2. J. Allergy Clin. Immunol. 139, 519-532 (2017).

143. Kim, R. Y. et al. Role for NLRP3 Inflammasome-mediated, IL-1 beta-Dependent Responses in Severe, Steroid-Resistant Asthma. Am. J. Respir. Crit. Care. Med. 196, 283-297 (2017).

144. Moffatt, M. F. et al. A large-scale, consortium-based genomewide association study of asthma. N. Engl. J. Med. 363, 1211-1221 (2010).

145. Stadhouders R. et al. Epigenome analysis links gene regulatory elements in group 2 innate lymphocytes to asthma susceptibility. J. Allergy Clin. Immunol. 142, 1793-1807 (2018).

146. Christianson, C. A. et al. Persistence of asthma requires multiple feedback circuits involving type 2 innate lymphoid cells and IL-33. J. Allergy Clin. Immunol. 136, 59-68.e14 (2015).

147. Smith, S. G. et al. Increased numbers of activated group 2 innate lymphoid cells in the airways of patients with severe asthma and persistent airway eosinophilia. J. Allergy Clin. Immunol. 137, 75-86.e78 (2016).

148. Hirose, K., Iwata, A., Tamachi, T. \& Nakajima, H. Allergic airway inflammation: key players beyond the Th2 cell pathway. Immunol. Rev. 278, 145-161 (2017).

149. Bartemes, K. R., Kephart, G. M., Fox, S. J. \& Kita, H. Enhanced innate type 2 immune response in peripheral blood from patients with asthma. J. Allergy Clin. Immunol. 134, 671-678.e674 (2014).

150. Martinez-Gonzalez, l. et al. Allergen-Experienced Group 2 Innate Lymphoid Cells Acquire Memory-like Properties and Enhance Allergic Lung Inflammation. Immunity 45, 198-208 (2016).

151. Barnig, C. et al. Lipoxin A4 regulates natural killer cell and type 2 innate lymphoid cell activation in asthma. Sci. Transl. Med. 5, 174ra126 (2013).

152. Britanova, L. \& Diefenbach, A. Interplay of innate lymphoid cells and the microbiota. Immunol. Rev. 279, 36-51 (2017).

153. Monticelli, L. A. et al. Arginase 1 is an innate lymphoid-cell-intrinsic metabolic checkpoint controlling type 2 inflammation. Nat. Immunol. 17, 656-665 (2016).

154. Essilfie, A. T. et al. Macrolide therapy suppresses key features of experimental steroid-sensitive and steroid-insensitive asthma. Thorax 70, 458-467 (2015).

155. Gauvreau, G. M. et al. Effects of an anti-TSLP antibody on allergen-induced asthmatic responses. N. Engl. J. Med. 370, 2102-2110 (2014).

156. Erpenbeck, V. J. et al. The oral CRTh2 antagonist QAW039 (fevipiprant): A phase II study in uncontrolled allergic asthma. Pulm. Pharmacol. Ther. 39, 54-63 (2016).

157. Kearley, J. et al. Cigarette smoke silences innate lymphoid cell function and facilitates an exacerbated type I interleukin-33-dependent response to infection. Immunity 42, 566-579 (2015).

158. Donovan C. et al. Roles for T/B lymphocytes and ILC2s in experimental chronic obstructive pulmonary disease. J. Leukoc. Biol. 105, 143-150 (2018).

159. Beckett, E. L. et al. A new short-term mouse model of chronic obstructive pulmonary disease identifies a role for mast cell tryptase in pathogenesis. J. Allergy Clin. Immunol. 131, 752-762 (2013).

160. Hsu, A. C. et al. Targeting PI3K-p110alpha Suppresses Influenza Virus Infection in Chronic Obstructive Pulmonary Disease. Am. J. Respir. Crit. Care. Med. 191, 1012-1023 (2015).

161. Hsu, A. C. et al. MicroRNA-125a and -b inhibit A20 and MAVS to promote inflammation and impair antiviral response in COPD. JCI Insight 2, e90443 (2017). 
162. Li, D. et al. IL-33 promotes ST2-dependent lung fibrosis by the induction of alternatively activated macrophages and innate lymphoid cells in mice. J. Allergy Clin. Immunol. 134, 1422-1432.e1411 (2014).

163. Hams, E. et al. IL-25 and type 2 innate lymphoid cells induce pulmonary fibrosis. Proc. Natl. Acad. Sci. USA 111, 367-372 (2014).

164. Vannella, K. M. et al. Combinatorial targeting of TSLP, IL-25, and IL-33 in type 2 cytokine-driven inflammation and fibrosis. Sci. Transl. Med. 8, 337ra365 (2016).

165. Moretti, S. et al. A mast cell-ILC2-Th9 pathway promotes lung inflammation in cystic fibrosis. Nat. Commun. 8, 14017 (2017).

166. Nausch N., Mutapi F. Group 2 ILCs: A way of enhancing immune protection against human helminths? Parasite Immunol. 40, (2018).

167. Hong, J. Y. et al. Neonatal rhinovirus induces mucous metaplasia and airways hyperresponsiveness through IL-25 and type 2 innate lymphoid cells. J. Allergy Clin. Immunol. 134, 429-439 (2014).

168. Han, M. et al. IFN-gamma Blocks Development of an Asthma Phenotype in Rhinovirus-Infected Baby Mice by Inhibiting Type 2 Innate Lymphoid Cells. Am. J. Respir. Cell Mol. Biol. 56, 242-251 (2017).

169. Rajput, C. et al. RORalpha-dependent type 2 innate lymphoid cells are required and sufficient for mucous metaplasia in immature mice. Am. J. Physiol. Lung Cell. Mol. Physiol. 312, L983-1993 (2017).

170. Han, M. et al. The Innate Cytokines IL-25, IL-33, and TSLP Cooperate in the Induction of Type 2 Innate Lymphoid Cell Expansion and Mucous Metaplasia in Rhinovirus-Infected Immature Mice. J. Immunol. (Baltim., Md: 1950) 199, 1308-1318 (2017)

171. Jackson, D. J. et al. IL-33-dependent type 2 inflammation during rhinovirusinduced asthma exacerbations in vivo. Am. J. Respir. Crit. Care. Med. 190, 1373-1382 (2014).

172. Stier, M. T. et al. Respiratory syncytial virus infection activates IL-13-producing group 2 innate lymphoid cells through thymic stromal lymphopoietin. J. Allergy Clin. Immunol. 138, 814-824.e811 (2016).

173. Califano, D. et al. IFN-gamma increases susceptibility to influenza A infection through suppression of group II innate lymphoid cells. Mucosal Immunol. 11, 209-219 (2018).

174. Chang, Y. J. et al. Innate lymphoid cells mediate influenza-induced airway hyperreactivity independently of adaptive immunity. Nat. Immunol. 12, 631-638 (2011).

175. Gorski, S. A., Hahn, Y. S. \& Braciale, T. J. Group 2 innate lymphoid cell production of IL-5 is regulated by NKT cells during influenza virus infection. PLoS. Pathog. 9, e1003615 (2013).

176. Oliphant, C. J. et al. MHCII-mediated dialog between group 2 innate lymphoid cells and CD4(+) T cells potentiates type 2 immunity and promotes parasitic helminth expulsion. Immunity 41, 283-295 (2014).

177. Molofsky, A. B. et al. Innate lymphoid type 2 cells sustain visceral adipose tissue eosinophils and alternatively activated macrophages. J. Exp. Med. 210, 535-549 (2013).

178. Walker, J. A. et al. Bcl11b is essential for group 2 innate lymphoid cell development. J. Exp. Med. 212, 875-882 (2015).

179. Li, B. W. et al. T cells are necessary for ILC2 activation in house dust miteinduced allergic airway inflammation in mice. Eur. J. Immunol. 46, 1392-1403 (2016).

180. Li, B. W. S., Beerens, D., Brem, M. D. \& Hendriks, R. W. Characterization of Group 2 Innate Lymphoid Cells in Allergic Airway Inflammation Models in the Mouse. Methods Mol. Biol. (Clifton, NJ) 1559, 169-183 (2017).

181. Hung, L. Y. et al. IL-33 drives biphasic IL-13 production for noncanonical Type 2 immunity against hookworms. Proc. Natl. Acad. Sci. USA 110, 282-287 (2013).

182. Doherty, T. A. et al. Lung type 2 innate lymphoid cells express cysteinyl leukotriene receptor 1 , which regulates $\mathrm{TH} 2$ cytokine production. J. Allergy Clin. Immunol. 132, 205-213 (2013).

183. Beale, J. et al. Rhinovirus-induced IL-25 in asthma exacerbation drives type 2 immunity and allergic pulmonary inflammation. Sci. Transl. Med 6, 256 ra134 (2014).

184. Shin, H. W. et al. IL-25 as a novel therapeutic target in nasal polyps of patients with chronic rhinosinusitis. J. Allergy Clin. Immunol. 135, 1476-1485.e1477 (2015).

185. Ballantyne, S. J. et al. Blocking IL-25 prevents airway hyperresponsiveness in allergic asthma. J. Allergy Clin. Immunol. 120, 1324-1331 (2007).
186. Scott, I. C., Houslay, K. F. \& Cohen, E. S. Prospects to translate the biology of IL-33 and ST2 during organ transplantation into therapeutics to treat graft-versushost disease. Ann. Transl. Med. 4, 500 (2016).

187. Corren, J. et al. Tezepelumab in Adults with Uncontrolled Asthma. N. Engl. J. Med. 377, 936-946 (2017).

188. Antoniu, S. A. MEDI-528, an anti-IL-9 humanized antibody for the treatment of asthma. Curr. Opin. Mol. Ther. 12, 233-239 (2010).

189. Hall, I. P. et al. Efficacy of $\mathrm{BI} 671800$, an oral $\mathrm{CRTH} 2$ antagonist, in poorly controlled asthma as sole controller and in the presence of inhaled corticosteroid treatment. Pulm. Pharmacol. Ther. 32, 37-44 (2015).

190. Barnes, N. et al. A randomized, double-blind, placebo-controlled study of the CRTH2 antagonist OC000459 in moderate persistent asthma. Clin. Exp. Allergy.: J. Br. Soc. Allergy. Clin. Immunol. 42, 38-48 (2012).

191. Kuna, P., Bjermer, L. \& Tornling, G. Two Phase II randomized trials on the CRTh2 antagonist AZD1981 in adults with asthma. Drug Des. Dev. Ther. 10, 2759-2770 (2016).

192. Gonem, S. et al. Fevipiprant, a prostaglandin D2 receptor 2 antagonist, in patients with persistent eosinophilic asthma: a single-centre, randomised, double-blind, parallel-group, placebo-controlled trial. Lancet Respir. Med 4, 699-707 (2016)

193. Huang, T. et al. Depletion of major pathogenic cells in asthma by targeting CRTh2. JCl Insight 1, e86689 (2016).

194. Xue, L. et al. Prostaglandin D2 activates group 2 innate lymphoid cells through chemoattractant receptor-homologous molecule expressed on $\mathrm{TH} 2$ cells. J. Allergy Clin. Immunol. 133, 1184-1194 (2014).

195. Bratt, J. M., Zeki, A. A., Last, J. A. \& Kenyon, N. J. Competitive metabolism of Larginine: arginase as a therapeutic target in asthma. J. Biomed. Res. 25, 299-308 (2011).

196. Maarsingh, H. et al. Arginase inhibition protects against allergen-induced airway obstruction, hyperresponsiveness, and inflammation. Am. J. Respir. Crit. Care Med. 178, 565-573 (2008).

197. Meurs, H. et al. Increased arginase activity underlies allergen-induced deficiency of cNOS-derived nitric oxide and airway hyperresponsiveness. Br. J. Pharmacol. 136, 391-398 (2002).

198. van den Berg, M. P., Meurs, H. \& Gosens, R. Targeting arginase and nitric oxide metabolism in chronic airway diseases and their co-morbidities. Curr. Opin. Pharmacol. 40, 126-133 (2018).

199. Sel, S. et al. Effective prevention and therapy of experimental allergic asthma using a GATA-3-specific DNAzyme. J. Allergy Clin. Immunol. 121, 910-916.e915 (2008).

200. Garn, H. \& Renz, H. GATA-3-specific DNAzyme - A novel approach for stratified asthma therapy. Eur. J. Immunol. 47, 22-30 (2017).

201. Krug, N. et al. Allergen-induced asthmatic responses modified by a GATA3specific DNAzyme. N. Engl. J. Med. 372, 1987-1995 (2015)

202. Wenzel, S. et al. Dupilumab efficacy and safety in adults with uncontrolled persistent asthma despite use of medium-to-high-dose inhaled corticosteroids plus a long-acting beta 2 agonist: a randomised double-blind placebo-controlled pivotal phase 2b dose-ranging trial. Lancet (Lond., Engl.) 388, 31-44 (2016).

203. Simpson, E. L. et al. Two Phase 3 Trials of Dupilumab versus Placebo in Atopic Dermatitis. N. Engl. J. Med. 375, 2335-2348 (2016).

204. Ortega, H. G. et al. Mepolizumab treatment in patients with severe eosinophilic asthma. N. Engl. J. Med. 371, 1198-1207 (2014)

205. Pavord, I. D. et al. Mepolizumab for severe eosinophilic asthma (DREAM): a multicentre, double-blind, placebo-controlled trial. Lancet (Lond., Engl.) 380, 651-659 (2012).

206. Busse, W. W., Ring, J., Huss-Marp, J. \& Kahn, J. E. A review of treatment with mepolizumab, an anti-IL-5 mAb, in hypereosinophilic syndromes and asthma. J. Allergy Clin. Immunol. 125, 803-813 (2010).

207. Rothenberg, M. E. et al. Treatment of patients with the hypereosinophilic syndrome with mepolizumab. N. Engl. J. Med. 358, 1215-1228 (2008).

208. Brightling, C. E. et al. Efficacy and safety of tralokinumab in patients with severe uncontrolled asthma: a randomised, double-blind, placebo-controlled, phase $2 \mathrm{~b}$ trial. Lancet Respir. Med. 3, 692-701 (2015).

209. Hanania, N. A. et al. Efficacy and safety of lebrikizumab in patients with uncontrolled asthma (LAVOLTA I and LAVOLTA II): replicate, phase 3, randomised, double-blind, placebo-controlled trials. Lancet Respir. Med. 4, 781-796 (2016). 